\title{
Integration of Cocycles and Lefschetz Number Formulae for Differential Operators
}

\author{
Ajay C. RAMADOSS \\ Department Mathematik, ETH Zürich, Rämistrasse 101, 8092 Zürich, Switzerland \\ E-mail: ajay.ramadoss@math.ethz.ch
}

Received August 12, 2010, in final form January 07, 2011; Published online January 18, 2011 doi:10.3842/SIGMA.2011.010

\begin{abstract}
Let $\mathcal{E}$ be a holomorphic vector bundle on a complex manifold $X$ such that $\operatorname{dim}_{\mathbb{C}} X=n$. Given any continuous, basic Hochschild $2 n$-cocycle $\psi_{2 n}$ of the algebra Diff ${ }_{n}$ of formal holomorphic differential operators, one obtains a $2 n$-form $f_{\mathcal{E}, \psi_{2 n}}(\mathcal{D})$ from any holomorphic differential operator $\mathcal{D}$ on $\mathcal{E}$. We apply our earlier results [J. Noncommut. Geom. 2 (2008), 405-448; J. Noncommut. Geom. 3 (2009), 27-45] to show that $\int_{X} f_{\mathcal{E}, \psi_{2 n}}(\mathcal{D})$ gives the Lefschetz number of $\mathcal{D}$ upto a constant independent of $X$ and $\mathcal{E}$. In addition, we obtain a "local" result generalizing the above statement. When $\psi_{2 n}$ is the cocycle from [Duke Math. J. 127 (2005), 487-517], we obtain a new proof as well as a generalization of the Lefschetz number theorem of Engeli-Felder. We also obtain an analogous "local" result pertaining to B. Shoikhet's construction of the holomorphic noncommutative residue of a differential operator for trivial vector bundles on complex parallelizable manifolds. This enables us to give a rigorous construction of the holomorphic noncommutative residue of $\mathcal{D}$ defined by B. Shoikhet when $\mathcal{E}$ is an arbitrary vector bundle on an arbitrary compact complex manifold $X$. Our local result immediately yields a proof of a generalization of Conjecture 3.3 of [Geom. Funct. Anal. 11 (2001), 1096-1124].
\end{abstract}

Key words: Hochschild homology; Lie algebra homology; Lefschetz number; Fedosov connection; trace density; holomorphic noncommutative residue

2010 Mathematics Subject Classification: 16E40; 32L05; 32C38; 58J42

\section{Introduction}

1.1. Let $X$ be a connected compact complex manifold such that $\operatorname{dim}_{\mathbb{C}} X=n$. Let $\mathcal{E}$ be a holomorphic vector bundle on $X$. In this paper, the term "vector bundle" shall hence forth mean "holomorphic vector bundle". Let $\mathcal{D} \operatorname{iff}(\mathcal{E})$ denote the sheaf of holomorphic differential operators on $\mathcal{E}$. Let $\Omega_{X}^{0, \bullet}$ denote the Dolbeault resolution of the sheaf $\mathcal{O}_{X}$ of holomorphic functions on $X$. Let $\operatorname{Diff}^{\bullet}(\mathcal{E})=\Omega_{X}^{0, \bullet} \otimes \mathcal{O}_{X} \operatorname{Diff}(\mathcal{E})$ and let $\operatorname{Diff}^{\bullet}(\mathcal{E})=\Gamma\left(X, \mathcal{D}\right.$ iff $\left.\bullet^{\bullet}(\mathcal{E})\right)$. There is a suitable topology on $\operatorname{Diff}^{\bullet}(\mathcal{E})$ which we describe in Section 2. Recall that any global holomorphic differential operator $\mathcal{D}$ induces an endomorphism of the Dolbeault complex $\mathrm{K}_{\mathcal{E}}{ }_{\mathcal{E}}:=\Gamma\left(X, \Omega_{X}^{0, \bullet} \otimes_{\mathcal{O}_{X}} \mathcal{E}\right)$ that commutes with the $\bar{\partial}$ differential. The Lefschetz number of $\mathcal{D}$, also known as the supertrace of $\mathcal{D}$,

$$
\operatorname{str}(\mathcal{D}):=\left.\sum_{i}(-1)^{i} \operatorname{Tr}(\mathcal{D})\right|_{\mathrm{H}^{i}(X, \mathcal{E})}
$$

therefore makes sense. More generally, let $\mathcal{E}$ be a vector bundle with bounded geometry on an arbitrary complex manifold $X$ of complex dimension $n$. The reader may refer to Section 2.2

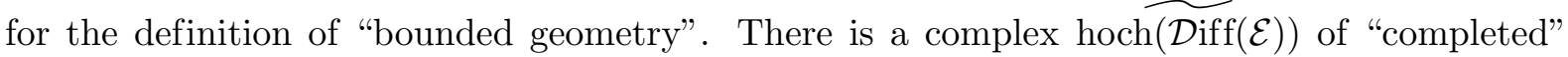
Hochschild chains that one can associate with $\mathcal{D} \operatorname{iff}^{\bullet}(\mathcal{E})$. This is a complex of (c-soft) sheaves of $\mathbb{C}$-vector spaces on $X$. Let $\Gamma_{c}$ denote "sections with compact support". Suppose that $\alpha \in$ $\Gamma_{c}(X, \operatorname{hoch}(\mathcal{D} \operatorname{iff}(\mathcal{E})))$ (see Section 2 for precise definitions). Let $\alpha_{0}$ denote the component of $\alpha$ 
with antiholomorphic degree 0 . For any $t>0, \alpha_{0} \mathrm{e}^{-t \Delta_{\mathcal{E}}}$ is a trace class operator on the (graded) Hilbert space $\mathrm{K}_{\mathcal{E} L^{2}}$ of square integrable elements of $\mathrm{K}_{\mathcal{E}}$ (Theorem 4, part 1). The supertrace $\operatorname{str}\left(\alpha_{0} e^{-t \Delta_{\mathcal{E}}}\right)$ of $\alpha_{0} \mathrm{e}^{-t \Delta_{\mathcal{E}}}$ is given by the formula

$$
\operatorname{str}\left(\alpha_{0} \mathrm{e}^{-t \Delta_{\mathcal{E}}}\right)=\left.\sum_{i}(-1)^{i} \operatorname{Tr}\left(\alpha_{0} \mathrm{e}^{-t \Delta_{\mathcal{E}}}\right)\right|_{\mathrm{K}_{\mathcal{E}, L^{2}}^{i}}
$$

We may define the Lefschetz number of $\alpha$ to be

$$
\lim _{t \rightarrow \infty} \operatorname{str}\left(\alpha_{0} e^{-t \Delta_{\mathcal{E}}}\right)
$$

When $X$ is compact and $\alpha=\mathcal{D}$ is a global holomorphic differential operator on $\mathcal{E}$, this coincides with the Lefschetz number of $\mathcal{D}$ defined earlier (any vector bundle on a compact manifold is of bounded geometry).

1.2. Let Diff $f_{n}$ denote the algebra $\mathbb{C}\left[\left[y_{1}, \ldots, y_{n}\right]\right]\left[\partial_{1}, \ldots, \partial_{n}\right]\left(\partial_{i}:=\frac{\partial}{\partial y_{i}}\right)$ of formal holomorphic differential operators. Denote the complex of continuous normalized Hochschild cochains of $\operatorname{Diff}_{n}$ with coefficients in Diff ${ }_{n}^{*}$ by $\mathrm{C}^{\bullet}\left(\operatorname{Diff}_{n}\right)$. Let $\psi_{2 n}$ be a cocycle in $\mathrm{C}^{2 n}\left(\operatorname{Diff}_{n}\right)$. For an algebra $A$, let $\mathrm{M}_{r}(A)$ denote the algebra of $r \times r$ matrices with entries in $A$. Then $\psi_{2 n}$ extends to a cocycle $\psi_{2 n}^{r}$ in $\mathrm{C}^{2 n}\left(\mathrm{M}_{r}\left(\operatorname{Diff}_{n}\right)\right)$ by the formula

$$
\psi_{2 n}^{r}\left(A_{0} \otimes D_{0}, \ldots, A_{2 n+1} \otimes D_{2 n+1}\right)=\operatorname{tr}\left(A_{0} \cdots A_{2 n+1}\right) \psi_{2 n}\left(D_{0}, \ldots, D_{2 n+1}\right) .
$$

Note that the group $G:=G L(n, \mathbb{C}) \times G L(r, \mathbb{C})$ acts on the algebra $\mathrm{M}_{r}\left(\operatorname{Diff}_{n}\right)$ with $G L(r, \mathbb{C})$ acting by conjugation and $G L(n, \mathbb{C})$ acting by linear coordinate changes. It can be checked without difficulty from (1) that if $\psi_{2 n}$ is $G L(n)$-basic, then $\psi_{2 n}^{r}$ is $G$-basic. We will define the term basic carefully in Section 3.2.2.

Given a basic Hochschild 2n-cocycle $\psi_{2 n}$, a Gel'fand-Fuks type procedure (which extends a construction from [10] and [17]) enables one to construct a map of complexes of sheaves

$$
\left.f_{\mathcal{E}, \psi_{2 n}}: \operatorname{hoch(\mathcal {Diff}}(\mathcal{E})\right) \rightarrow \Omega_{X}^{2 n-\bullet}
$$

where the target has the usual de Rham differential (see Section 3.2 for details). Our extension of the construction from [10, 17] is made possible by a result from [7] (Theorem 5) showing the existence of a "good" choice of Fedosov connection on the bundle $\mathcal{B}_{\mathcal{E}}$ of fibrewise formal differential operators on $\mathcal{E}$. $f_{\mathcal{E}, \psi_{2 n}}$ induces a map of complexes

$$
\left.f_{\mathcal{E}, \psi_{2 n}}: \quad \Gamma_{c}(X, \operatorname{hoch(\mathcal {Diff}}(\mathcal{E}))\right) \rightarrow \Omega_{c}^{2 n-\bullet}(X) .
$$

The subscript ' $c$ ' above indicates "compact supports". As a map of complexes of sheaves, $f_{\mathcal{E}, \psi_{2 n}}$ depends upon the choice of a Fedosov connection on $\mathcal{B}_{\mathcal{E}}$. Nevertheless, as a map in the bounded derived category $\mathrm{D}^{b}\left(\mathrm{Sh}_{\mathbb{C}}[X]\right)$ of sheaves of $\mathbb{C}$-vector spaces on $X, f_{\mathcal{E}, \psi_{2 n}}$ is independent of the choice of Fedosov connection on the bundle of fibrewise formal differential operators on $\mathcal{E}$ (see Proposition 15). Let $\tau_{2 n}$ denote the normalized Hochschild cocycle from [11]. Let [..] denote "class in cohomology". Since $\mathrm{HH}^{2 n}\left(\operatorname{Diff}_{n}\right)$ is 1-dimensional, the ratio $\frac{\left[\psi_{2 n}\right]}{\left[\tau_{2 n}\right]}$ makes sense.

1.3. One of the main results in this paper (proven in Section 3) is the following.

Theorem 1. If $\left.\alpha \in \Gamma_{c}(X, \widehat{\operatorname{hoch}(\mathcal{D i f f}}(\mathcal{E}))\right)$ is a 0 -cycle,

$$
\int_{X} f_{\mathcal{E}, \psi_{2 n}}(\alpha)=(2 \pi i)^{n} \frac{\left[\psi_{2 n}\right]}{\left[\tau_{2 n}\right]} \cdot \lim _{t \rightarrow \infty} \operatorname{str}\left(\alpha_{0} e^{-t \Delta_{\mathcal{E}}}\right) .
$$

A special case of the above theorem, when $\alpha=\mathcal{D}$ is a global holomorphic differential operator on $X$ and when $X$ is compact is as follows. 


\section{Theorem 2.}

$$
\int_{X} f_{\mathcal{E}, \psi_{2 n}}(\mathcal{D})=(2 \pi i)^{n} \frac{\left[\psi_{2 n}\right]}{\left[\tau_{2 n}\right]} \cdot \operatorname{str}(\mathcal{D})
$$

Theorem 1 is proven using results from [18] and [19]. If $\psi_{2 n}$ is known explicitly, $f_{\mathcal{E}}(\alpha)$ can in principle be locally calculated explicitly for $\left.\alpha \in \Gamma_{c}(X, \widehat{\operatorname{hoch}} \widetilde{(\mathcal{D i f f}}(\mathcal{E}))\right)$.

1.4 In particular, if we put $\psi_{2 n}$ to be the normalized Hochschild cocycle $\tau_{2 n}$ of [11], the $2 n$-form $f_{\mathcal{E}, \tau_{2 n}}(\mathcal{D})$ was denoted in $[10]$ by $\chi_{0}(\mathcal{D})$. We therefore obtain a different proof of the following Lefschetz number theorem of [10] as well as a generalization of this result.

Engeli-Felder theorem [10]. If $X$ is compact and $\mathcal{D} \in \Gamma(X, \mathcal{D} \operatorname{iff}(\mathcal{E}))$,

$$
\operatorname{str}(\mathcal{D})=\frac{1}{(2 \pi i)^{n}} \int_{X} \chi_{0}(\mathcal{D})
$$

We sketch in a remark Section 3.5 why the map $\alpha \mapsto \int_{X} f_{\mathcal{E}, \psi_{2 n}}(\alpha)$ may be viewed as an "integral of $\psi_{2 n}^{r}$ over $X$ " in some sense. Upto this point, this paper completes our work in [18] and [19] by combining the results therein with an extension of the trace density construction of [10] using a more delicate choice of Fedosov connection on $\mathcal{B}_{\mathcal{E}}$.

1.4.1. Let us briefly summarize our approach to Lefschetz number formulae for differential operators in $[18,19]$ and the current paper. Our approach is to show that the two sides of Theorem 1 coincide upto a constant fact $C\left(\psi_{2 n}\right)$ independent of $X$ and $\mathcal{E}$ such that $C\left(\psi_{2 n}\right)=$ $\frac{\left[\psi_{2 n}\right]}{\left[\tau_{2 n}\right]} C\left(\tau_{2 n}\right)$. Compared to the approach in [10], the analytic routine part of this step is minimal: beyond proving that $\alpha \mapsto \lim _{t \rightarrow \infty} \operatorname{str}\left(\alpha_{0} \mathrm{e}^{-t \Delta_{\mathcal{E}}}\right)$ vanishes on 0-boundaries (which required only the most basic heat kernel estimates (see [18])), this step requires a few simple, natural arguments from $[18,19]$ and the current paper. $C\left(\tau_{2 n}\right)$ can then be determined by computing both sides of Theorem 1 for the identity operator on $\left(\mathbb{P}^{1}\right)^{\times n}$ (see [19]). In particular, the local, more general result of Theorem 1 is an integral part of our approach and the Engeli-Felder formula follows as a direct corollary of this result. As far as this author can see, local results like Theorem 1 do not appear in the approach in [10].

1.5. We proceed to prove Conjecture 3.3 from [20] in greater generality. In [20], B. Shoikhet starts with a particular $2 n+1$ cocycle $\Psi_{2 n+1} \in \mathrm{C}_{\text {Lie }}^{2 n+1}\left(\mathfrak{g}_{\infty}^{\text {fin }}\left(\right.\right.$ Diff $\left.\left._{n}\right), \mathbb{C}\right)$ called a lifting formula. He then attempts to construct a map of complexes

$$
\Theta: \quad C_{\bullet}^{\text {lie }}\left(\mathfrak{g l}_{\infty}^{\mathrm{fin}}(\operatorname{Diff}(\mathcal{E})), \mathbb{C}\right) \rightarrow \Omega^{2 n+1-\bullet}(X) \text {. }
$$

For any global holomorphic differential operator $\mathcal{D}$ on $\mathcal{E}$, he refers to the number $\int_{X} \Theta\left(E_{11}(\mathcal{D})\right)$ as its holomorphic noncommutative residue (whenever $X$ is compact). Since $\Psi_{2 n+1}$ is not $G L(n)$ basic, this cannot be done as directly as in [20] unless $X$ is complex parallelizable and $\mathcal{E}$ is trivial (as per our understanding).

One can construct a complex of "completed" Lie chains $\widetilde{\operatorname{Lie}}(\mathcal{D} \operatorname{iff}(\mathcal{E}))$ of $\mathfrak{g} \mathfrak{l}_{\infty}^{\mathrm{fin}}(\mathcal{D} \operatorname{iff}(\mathcal{E})$ ) (see Section 2.4). This complex is analogous to $\widetilde{\operatorname{hoch}}(\mathcal{D} \operatorname{iff}(\mathcal{E})$ ). For a trivial vector bundle $\mathcal{E}$ (with bounded geometry) on a parallelizable (not necessarily compact) complex manifold $X$, we construct a map of complexes of sheaves

$$
\lambda\left(\Psi_{2 n+1}^{r}\right): \quad \widetilde{\operatorname{Lie}}(\mathcal{D} \operatorname{iff}(\mathcal{E}))[1] \rightarrow \Omega_{X}^{2 n-\bullet}
$$

using B. Shoikhet's lifting formulas. Let $\alpha \in \Gamma_{c}(X, \widetilde{\operatorname{Lie}}(\mathcal{D} \operatorname{iff}(\mathcal{E}))[1])$ be a nontrivial 0-cycle. The component $\alpha_{0}$ of $\alpha$ of antiholomorphic degree 0 is a finite matrix with entries in $\operatorname{Diff}^{0}(\mathcal{E})$. 


\section{Theorem 3.}

$$
\int_{X} \lambda\left(\Psi_{2 n+1}\right)(\alpha)=C \cdot \lim _{t \rightarrow \infty} \operatorname{str}\left(\operatorname{Tr}\left(\alpha_{0}\right) e^{-t \Delta_{\mathcal{E}}}\right),
$$

where $C$ is a constant independent of $X$ and $\mathcal{E}$.

We prove Theorem 3 in Section 4.2. The methods used for this are similar to those used in [18] and [19]. In Sections 4.2.5 and 4.2.6, we provide a rigorous (though not as explicit as we had originally hoped for) definition of B. Shoikhet's holomorphic noncommutative residue of a holomorphic differential operator $\mathcal{D}$ on $\mathcal{E}$ for the case when $\mathcal{E}$ is an arbitrary vector bundle over an arbitrary compact complex manifold $X$. Theorem 3 easily implies that this number is $C$ times the Lefschetz number of $\mathcal{E}$ ( $C$ being the constant from Theorem 3$)$. We also remark that if we replace $\Psi_{2 n+1}$ by a $G L(n)$-basic cocycle $\Theta_{2 n+1}$ representing the cohomology class [ $\left.\Psi_{2 n+1}\right]$, Theorem 3 holds in full generality (i.e., for arbitrary vector bundles with bounded geometry over complex manifolds that are not parallelizable). Indeed, the proof of Lemma 5.2 of [10] can be modified to show the existence of such a $G L(n)$-basic representative of $\left[\Psi_{2 n+1}\right]$. This settles Conjecture 3.3 of $[20]$.

Convention 0. We now state some conventions that stand throughout this paper.

(i) The term complex shall be understood to mean a complex with homological grading with one exception that we point out in (iv) below.

(ii) The term double complex will mean "complex of complexes". Let $\left\{C_{p, q}\right\}$ be a double complex with horizontal and vertical differentials $d_{h}$ and $d_{v}$ respectively. One associates two total complexes $\operatorname{Tot}^{\oplus}(C)$ and $\operatorname{Tot}^{\Pi}(C)$ with the double complex $C$, where

$$
\left(\operatorname{Tot}^{\oplus}(C)\right)_{n}:=\oplus_{p+q=n} C_{p, q}, \quad\left(\operatorname{Tot}^{\Pi}(C)\right)_{n}:=\Pi_{p+q=n} C_{p, q}
$$

The differential we use on the shifted total complexes $\operatorname{Tot}^{\oplus}(C)[k]$ and $\operatorname{Tot}^{\Pi}(C)[k]$ will satisfy $d(x)=d_{h}(x)+(-1)^{|x|} d_{v}(x)$ for any homogenous element $x$ of $\operatorname{Tot}(C)[k]$.

(iii) The DG-algebras that we work with naturally come with cohomological grading. If $A^{\bullet}$ is a (cohomologically graded) DG-algebra, we automatically view it as a homologically graded algebra by inverting degrees (i.e., elements of $A^{n}$ have chain degree $-n$ ). The notation $A^{\bullet}$ in this case is still continued in order not to introduce unnatural notation. The same principle holds for DG-modules over DG-algebras that we work with.

(iv) When computing the hypercohomology of a complex $\mathcal{F}_{\bullet}$ of sheaves on $X$, we convert $\mathcal{F}_{\bullet}$ into a complex with cohomological grading by inverting degrees.

(v) For a complex $\mathcal{F}_{\bullet}$ of sheaves on $X, \mathcal{H}_{\bullet}(\mathcal{F})$ shall denote the (graded) sheaf of homologies of $\mathcal{F}_{\bullet}$.

\section{The Hochschild chain complex $\Gamma(X, \operatorname{hoch} \widetilde{(\mathcal{D i f f}}(\mathcal{E})))$ and other preliminaries}

2.1. Let $\mathcal{D} \operatorname{iff} \bullet(\mathcal{E})(U)$ denote $\Gamma\left(U, \Omega_{X}^{0, \bullet} \otimes_{\mathcal{O}_{X}} \mathcal{D} \operatorname{iff}(\mathcal{E})\right)$. Let $A^{\bullet}$ be any DG-algebra. Let $\mathrm{C}_{\bullet}\left(A^{\bullet}\right)$ denote the complex of Hochschild chains of $A^{\bullet}$. Whenever necessary, $\mathrm{C}_{\bullet}\left(A^{\bullet}\right)$ will be converted to a cochain complex by inverting degrees. Note that the differential on $\mathrm{C}_{\bullet}\left(\mathcal{D i f f}^{\bullet}(\mathcal{E})(U)\right)$ extends to a differential on the graded vector space $\oplus_{k} \mathcal{D}$ iff ${ }^{\bullet}\left(\mathcal{E}^{\boxplus k}\right)\left(U^{k}\right)[k-1]$ where $\mathcal{E}^{\bigotimes k}$ denotes the vector bundle $\pi_{1}^{*} \mathcal{E} \otimes \cdots \otimes \pi_{k}^{*} \mathcal{E}$ on $X^{k}$ (with $\pi_{j}: X^{k} \rightarrow X$ denoting the projection to the $j$-th factor). We denote the resulting complex by $\widehat{\mathrm{C}_{\bullet}}(\mathcal{D}$ iff $\bullet(\mathcal{E})(U))$. 
Let hoch $\widetilde{\mathcal{D i f f}}(\mathcal{E}))$ denote the sheaf associated to the presheaf

$$
U \mapsto \widehat{\mathrm{C}_{\bullet}}\left(\mathcal{D} \operatorname{iff}^{\bullet}(\mathcal{E})(U)\right)
$$

of complexes of $\mathbb{C}$-vector spaces. Note that left multiplication of a homogenous element of $\mathcal{D}$ iff $f^{\bullet}(\mathcal{E})(U)$ by a smooth function on $U$ is well defined. It follows that each $\operatorname{Diff}^{\bullet}\left(\mathcal{E}^{\bowtie k}\right)\left(U^{k}\right)$ is a graded left $\mathrm{C}^{\infty}(U)$-module via

$$
f . D:=\pi_{1}^{*}(f) . D \quad \text { for all } f \in \mathrm{C}^{\infty}(U), \quad D \in \mathcal{D i f f}^{\bullet}\left(\mathcal{E}^{\bowtie k}\right)\left(U^{k}\right) .
$$

Hence, hoch $\widetilde{\mathcal{D} \operatorname{iff}}(\mathcal{E}))$ is a complex each of whose terms is a module over the sheaf of smooth functions on $X$ (though the differential is not compatible with this $C^{\infty}$-module structure). It follows that hoch( $\widetilde{\mathcal{D i f f}}(\mathcal{E}))$ is a complex of flabby sheaves on $X$. Therefore,

$$
\left.\left.\mathrm{H}_{\bullet}(\Gamma(X, \operatorname{hoch} \widetilde{\mathcal{D i f f}}(\mathcal{E})))\right) \simeq \mathbb{H}^{\bullet}(X, \operatorname{hoch(\mathcal {Diff}}(\mathcal{E}))\right) .
$$

Here, one must convert the chain complex hoch $\widetilde{(\mathcal{D i f f}}(\mathcal{E}))$ into a cochain complex by inverting degrees before taking hypercohomology.

\section{Proposition 1.}

$$
\left.\mathrm{H}_{i}(\Gamma(X, \operatorname{hoch} \widetilde{\mathcal{D} \operatorname{iff}}(\mathcal{E})))\right) \simeq \mathrm{H}^{2 n-i}(X, \mathbb{C}) \quad \text { whenever } \quad 0 \leq i \leq 2 n .
$$

Proof. Note that the differential on the Hochschild chain complex $\mathrm{C}_{\bullet}(\Gamma(U, \mathcal{D} \operatorname{iff}(\mathcal{E})))$ extends to a differential on the graded vector space $\oplus_{k} \Gamma\left(U^{k}, \mathcal{D} \operatorname{iff}\left(\mathcal{E}^{\nabla k}\right)\right)[k-1]$. Call the resulting complex $\widehat{\mathrm{C}_{\bullet}}(\Gamma(U, \mathcal{D} \operatorname{iff}(\mathcal{E})))$. One can then consider the sheaf $\widehat{\mathrm{C}_{\bullet}}(\mathcal{D} \operatorname{iff}(\mathcal{E}))$ associated to the presheaf

$$
U \mapsto \widehat{\mathrm{C}_{\bullet}}(\Gamma(U, \mathcal{D} \operatorname{iff}(\mathcal{E})))
$$

of complexes of $\mathbb{C}$-vector spaces. We claim that the natural map of complexes from $\widehat{\mathrm{C}_{\bullet}}(\mathcal{D} \operatorname{iff}(\mathcal{E}))$ to hoch $\widetilde{\operatorname{Diff}}(\mathcal{E}))$ is a quasiisomorphism. To prove our claim, it suffices to verify that the natural map of complexes from $\widehat{\mathrm{C}_{\bullet}}(\mathcal{D} \operatorname{iff}(\mathcal{E}))$ to hoch $\left.\widetilde{\mathcal{D} \operatorname{iff}}(\mathcal{E})\right)$ locally induces an isomorphism on cohomology sheaves. Indeed, on any open subset $U$ of $X$ on which $\mathcal{E}$ and $T_{X}^{1,0}$ are trivial, the natural map from $\Gamma\left(U^{k}, \mathcal{D} \operatorname{iff}\left(\mathcal{E}^{\square k}\right)\right)$ to $\Gamma\left(U^{k}, \mathcal{D} \operatorname{iff} \bullet\left(\mathcal{E}^{\square k}\right)\right)$ is a quasiisomorphism (since $\mathcal{O}_{X^{k}}$ is quasiisomorphic to $\left(\Omega_{X^{k}}^{0, \bullet}, \bar{\partial}\right)$ and $\Gamma\left(U^{k}, \mathcal{D} \operatorname{iff}\left(\mathcal{E}^{\bigotimes k}\right)\right)$ is a free module over $\left.\Gamma\left(U^{k}, \mathcal{O}_{X^{k}}\right)\right)$. The desired proposition then follows from $(2)$ and the fact that $\widehat{\mathrm{C}_{\bullet}}(\mathcal{D} \operatorname{iff}(\mathcal{E}))$ is quasiisomorphic to the shifted constant sheaf $\underline{\mathbb{C}}[2 n]$. This fact (see [18, Lemma 3]) is also implicit in many earlier papers, for instance, [5].

Convention 1. Whenever we identify $\mathrm{H}_{i}(\Gamma(X$, hoch $\widetilde{(\mathcal{D i f f}}(\mathcal{E})))$ with $\mathrm{H}^{2 n-i}(X, \mathbb{C})$, we use the identification coming from a specific quasiisomorphism between $\widehat{\mathrm{C}_{\bullet}}(\mathcal{D} \operatorname{iff}(\mathcal{E}))$ and $\underline{\mathbb{C}}[2 n]$. This quasiisomorphism is constructed as follows. "Morita invariance" yields a quasiisomorphism between $\widehat{\mathrm{C}_{\bullet}}(\mathcal{D} \operatorname{iff}(\mathcal{E}))$ and $\widehat{\mathrm{C}_{\bullet}}(\mathcal{D} \operatorname{iff}(X)$ ) (see the proof of Lemma 3 of [18]). By a result of [5], $\widehat{\mathrm{C}_{\bullet}}(\mathcal{D} \operatorname{iff}(X))$ is quasiisomorphic to $\mathbb{C}[2 n]$. The quasiisomorphism we shall use throughout this paper is the one taking the class of the normalized Hochschild $2 n$-cycle

$$
\sum_{\sigma \in S_{2 n}} \operatorname{sgn}(\sigma)\left(1, z_{1}, \frac{\partial}{\partial z_{1}}, \ldots, z_{n}, \frac{\partial}{\partial z_{n}}\right)
$$

to $1[2 n]$ on any open subset $U$ of $X$ with local holomorphic coordinates $z_{1}, \ldots, z_{n}$. We remark that in the above formula, any permutation $\sigma$ in $S_{2 n}$ permutes the last $2 n$ factors leaving the first one fixed. We denote this identification by $\beta_{\mathcal{E}}$ as we did in [18]. 
2.1.1. As in [18], we define a topology on $\operatorname{Diff}^{\bullet}(\mathcal{E})$ as follows. Let $\operatorname{Diff} \leq k, \bullet(\mathcal{E})$ denote $\Gamma\left(X, \Omega_{X}^{0, \bullet} \otimes \mathcal{D i f f}^{\leq k}(\mathcal{E})\right)$ where $\mathcal{D i f f}^{\leq k}(\mathcal{E})$ denotes the sheaf of holomorphic differential operators on $\mathcal{E}$ of order $\leq k$. Equip $\mathcal{E}$ and $\Omega_{X}^{0, \bullet}$ with Hermitian metrics. Equip $\operatorname{Diff} \leq k, \bullet(\mathcal{E})$ with the topology generated by the family of seminorms $\left\{\|\cdot\|_{\phi, K, s} \mid K \subset X\right.$ compact, $s \in \Gamma\left(K, \mathcal{E} \otimes \Omega^{\bullet}\right)$, $\phi$ a $\mathrm{C}^{\infty}$ differential operator on $\mathcal{E}$ \} given by

$$
\|D\|_{\phi, K, s}=\operatorname{Sup}\{\|\phi D(s)(x)\| \mid x \in K\}
$$

The topology on $\operatorname{Diff}^{\bullet}(\mathcal{E})$ is the direct limit of the topologies on the $\operatorname{Diff} \leq k, \bullet(\mathcal{E})$. More generally, for any open subset $U$ of $X$, one can define a topology on $\Gamma\left(U, \mathcal{D}\right.$ iff $\left.f^{\bullet}(\mathcal{E})\right)$ in the same way.

2.2. Let $\mathcal{E}$ be a holomorphic vector bundle on an arbitrary connected complex manifold $X$. Let $\Delta_{\mathcal{E}}$ denote the Laplacian of $\mathcal{E}$ (for the operator $\bar{\partial}_{\mathcal{E}}$ ). This depends on the choice of Hermitian metric for $\mathcal{E}$ as well as on a choice of a Hermitian metric for $X$. Recall that $\Delta_{\mathcal{E}}=\Delta^{\mathcal{E}}+F$ where $\Delta^{\mathcal{E}}$ is the Laplacian of a $\mathrm{C}^{\infty}$-connection on $\mathcal{E}$ (see Definition 2.4 of [2]) and $F \in \Gamma(X, \operatorname{End}(\mathcal{E})$ ).

Definition 1. We say that $\mathcal{E}$ has bounded geometry if for some choice of Hermitian metric on $\mathcal{E}$, there exists a connection $\nabla_{\mathcal{E}}$ on $\mathcal{E}$ such that $\Delta_{\mathcal{E}}=\Delta^{\mathcal{E}}+F$, where $\Delta^{\mathcal{E}}$ is the Laplacian of $\nabla_{\mathcal{E}}$ and $F \in \Gamma(X, \operatorname{End}(\mathcal{E}))$, and all covariant derivatives of the curvature of $\nabla_{\mathcal{E}}$ as well as those of $F$ are bounded on $X$. In particular, any holomorphic vector bundle on a compact complex manifold has bounded geometry.

Let $\mathcal{E}$ be a vector bundle with bounded geometry on $X$. Let $\mathrm{K}^{\bullet}{ }_{\mathcal{E}} L^{2}$ denote the Hilbert space of square integrable sections of $\mathrm{K}^{\bullet} \mathcal{E}$. Then, $\mathrm{e}^{-t \Delta_{\mathcal{E}}}$ makes sense as an integral operator on $\mathrm{K}_{\mathcal{E}} L^{2}$ for any $t>0$ (see [9]). Let $\Gamma_{c}$ denote the functor "sections with compact support". Suppose that $\left.\alpha \in \Gamma_{c}(X, \operatorname{hoch(\mathcal {Diff}}(\mathcal{E}))\right)$ is a 0 -chain. Let $\alpha_{0}$ denote the component of $\alpha$ with antiholomorphic degree 0 . We recall the main results of [18] and [19] in the following theorem.

\section{Theorem 4 .}

(1) For any 0 -chain $\alpha, \alpha_{0} e^{-t \Delta_{\mathcal{E}}}$ is a trace class operator on $\mathrm{K}_{\mathcal{E} L^{2}}$ for any $t>0$.

(2) Further, if $\alpha$ is a 0 -cycle,

$$
\lim _{t \rightarrow \infty} \operatorname{str}\left(\alpha_{0} \mathrm{e}^{-t \Delta_{\mathcal{E}}}\right)=\frac{1}{(2 \pi i)^{n}} \int_{X}[\alpha]
$$

where $[\alpha]$ denotes the class of $\alpha$ in $\mathrm{H}_{c}^{2 n}(X, \mathbb{C})$.

Remark 1. Let us recall some aspects of [18] and [19]. When $X$ is compact, the linear functional

$$
\alpha \mapsto \lim _{t \rightarrow \infty} \operatorname{str}\left(\alpha_{0} \mathrm{e}^{-t \Delta_{\mathcal{E}}}\right)
$$

on $\left.\Gamma_{c}(X, \operatorname{hoch} \widetilde{(\mathcal{D i f f}}(\mathcal{E}))\right)$ is really an extension of the Feigin-Losev-Shoikhet-Hochschild 0-cocycle of $\left.\operatorname{Diff} f^{\bullet}\right)$. We therefore, denote the linear functional given by $(3)$ by $I_{\mathrm{FLS}}^{\text {hoch }}$. To show that the Feigin-Losev-Shoikhet cocycle indeed gives a linear functional on $\mathrm{H}_{0}(\Gamma(X, \operatorname{hoch}(\widetilde{\mathcal{D i f f}}(\mathcal{E}))))$ takes the sole computational effort in this whole program. The crux of this is a certain "estimate" of the Feigin-Losev-Shoikhet cocycle (Proposition 3 of [18]). This estimate is proven in "greater than usual" detail in [18]. It is done using nothing more than the simplest heat kernel estimates (Proposition 2.37 in [2]).

The second part of Theorem 4 can be exploited to extend the Feigin-Losev-Shoikhet cocycle to a linear functional on certain homology theories that are closely related to hoch( $\widetilde{\mathcal{D i f f}}(\mathcal{E}))$.

2.3. Cyclic homology. One obtains a "completed" version of the Bar complex of $\Gamma\left(U, \mathcal{D} \operatorname{iff}^{\bullet}(\mathcal{E})\right)$ by replacing $\Gamma\left(U, \mathcal{D} \text { iff } \bullet^{\bullet}(\mathcal{E})\right)^{\otimes k}$ by $\Gamma\left(U^{k}, \mathcal{D}\right.$ iff $\left.\bullet^{\bullet}\left(\mathcal{E}^{\bowtie k}\right)\right)$. Sheafification then gives 
us a complex bar $\widetilde{(\mathcal{D i f f}}(\mathcal{E}))$ of sheaves on $X$ having the same underlying graded vector space as hoch $\widetilde{\mathcal{D i f f}}(\mathcal{E}))$. One can then construct Tsygan's double complex

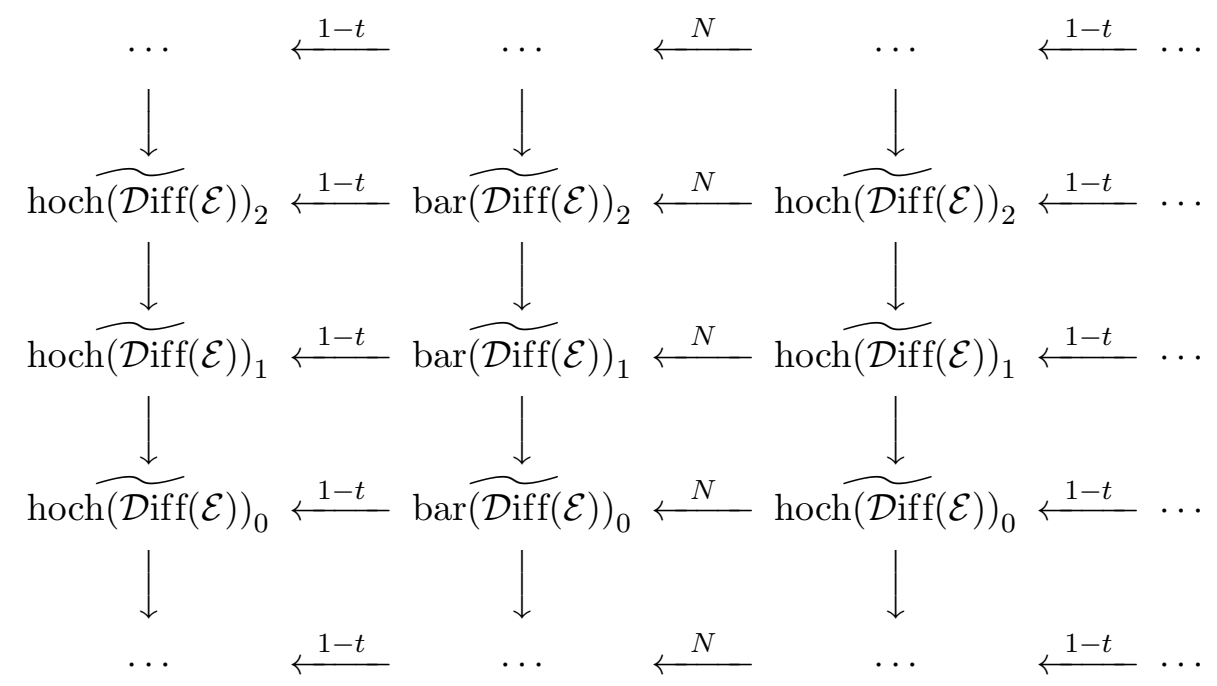

The total complex of this double complex is denoted by $\operatorname{Cycl} \widetilde{(\mathcal{D i f f}}(\mathcal{E}))$. Here, the operator $t$ acts on $\Gamma\left(U, \mathcal{D} \operatorname{iff}^{\bullet}(\mathcal{E})\right)^{\otimes k}$ by

$$
D_{1} \otimes \cdots \otimes D_{k} \mapsto(-1)^{k}(-1)^{d_{k}\left(d_{1}+\cdots+d_{k-1}\right)} D_{k} \otimes D_{1} \otimes \cdots \otimes D_{k-1},
$$

where the $D_{i}$ are homogenous elements of $\Gamma\left(U, \mathcal{D}\right.$ iff $\left.f^{\bullet}(\mathcal{E})\right)$ of degree $d_{i}$. $t$ then extends to $\Gamma\left(U^{k}, \mathcal{D i f f}^{\bullet}\left(\mathcal{E}^{凶 k}\right)\right)$ by continuity. It further extends to a map from $\left.\operatorname{bar} \widetilde{(\mathcal{D i f f}}(\mathcal{E})\right)$ to hoch $\left.\widetilde{\mathcal{D i f f}}(\mathcal{E})\right)$ by sheafification. Similarly, $N$ is the extension of the endomorphism $1+t+t^{2}+\cdots+t^{k-1}$ of

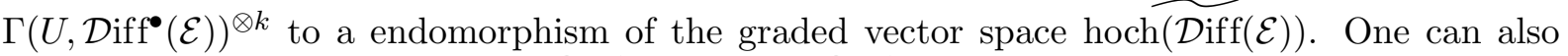
consider the Connes complex $\operatorname{Co} \widetilde{(\mathcal{D i f f}}(\mathcal{E})):=\operatorname{hoch} \widetilde{(\mathcal{D i f f}}(\mathcal{E})) /(1-t)$. There is a natural surjection $\Pi: \operatorname{Cycl}(\widetilde{\mathcal{D i f f}}(\mathcal{E})) \rightarrow \operatorname{Co} \widetilde{(\mathcal{D i f f}(\mathcal{E})})$ given by the quotient map on the first column of the double complex (4) and 0 on other columns. We omit the proof of the following standard proposition.

Proposition 2. П is a quasi-isomorphism.

Applying the linear functional $I_{\mathrm{FLS}}^{\text {hoch }}$ on the first column of the double complex of compactly supported global sections of the double complex (4), one obtains a linear functional $I_{\mathrm{FLS}}^{\mathrm{Cycl}}$ on $\left.\Gamma_{c}(X, \operatorname{Cycl} \widetilde{(\mathcal{D i f f}}(\mathcal{E}))\right)$. Moreover, the endomorphism $1-t$ of the vector space

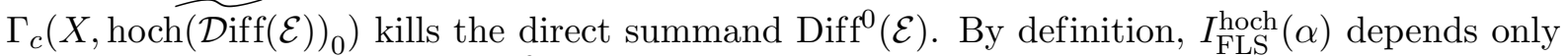
on the component of $\alpha$ in $\operatorname{Diff}^{0}(\mathcal{E})$. It follows that $I_{\mathrm{FLS}}^{\text {hoch }}$ vanishes on the image of $1-t$. It therefore, induces a linear functional on $\Gamma_{c}(X, \widehat{\operatorname{Co} \widetilde{(\mathcal{D i f f}}(\mathcal{E}))})$ which we will denote by $I_{\mathrm{FLS}}^{\mathrm{Co}}$. More explicitly, if $\alpha$ is a 0 -chain in $\Gamma_{c}(X, \widehat{\operatorname{Co}(\widetilde{\mathcal{D i f f}}(\mathcal{E}))}), I_{\mathrm{FLS}}^{\mathrm{Co}}(\alpha)=\lim _{t \rightarrow \infty} \operatorname{str}\left(\alpha_{0} \mathrm{e}^{-t \Delta_{\mathcal{E}}}\right)$ where $\alpha_{0}$ is the component of $\alpha$ in $\operatorname{Diff}^{0}(\mathcal{E})$. The following proposition is a consequence of part 2 of Theorem 4 .

Proposition 3. $I_{\mathrm{FLS}}^{\mathrm{Cycl}}$ and $I_{\mathrm{FLS}}^{\mathrm{Co}}$ descend to linear functionals on $\left.\mathrm{H}_{0}\left(\Gamma_{c}(X, \operatorname{Cycl} \widetilde{(\mathcal{D i f f}}(\mathcal{E}))\right)\right)$ and $\mathrm{H}_{0}\left(\Gamma_{c}(X, \operatorname{Cycl}(\mathcal{D i f f}(\mathcal{E})))\right)$ respectively.

Proof. We have already observed that $I_{\mathrm{FLS}}^{\text {hoch }}$ vanishes on the image of $1-t$ in $\Gamma_{c}\left(X, \operatorname{hoch}(\mathcal{D i f f}(\mathcal{E}))_{0}\right)$. That it also vanishes on the image of the Hochschild boundary $b$ of $\Gamma_{c}(X, \operatorname{hoch}(\widetilde{\mathcal{D i f f}}(\mathcal{E})))$ is implied by part 2 of Theorem 4 . The desired proposition then follows from the construction of $I_{\mathrm{FLS}}^{\mathrm{Cycl}}$ and $I_{\mathrm{FLS}}^{\mathrm{Co}}$. 
We recall from [19] that

$$
\left.\mathrm{H}_{0}\left(\Gamma_{c}(X, \operatorname{Cycl} \widetilde{(\mathcal{D i f f}}(\mathcal{E}))\right)\right) \simeq \mathrm{H}^{2 n}(X, \mathbb{C}) .
$$

It follows from Proposition 2 that

$$
\mathrm{H}_{0}\left(\Gamma_{c}(X, \widehat{\operatorname{Co} \widetilde{(\mathcal{D i f f}}(\mathcal{E}))})\right) \simeq \mathrm{H}^{2 n}(X, \mathbb{C})
$$

as well. Denote the natural projection from $\left.\Gamma_{c}(X, \operatorname{hoch} \widetilde{(\mathcal{D i f f}}(\mathcal{E}))\right)$ to $\left.\Gamma_{c}(X, \operatorname{Co} \widetilde{(\mathcal{D i f f}(\mathcal{E})})\right)$ by $p$. The following proposition is immediate from the definition of $I_{\mathrm{FLS}}^{\mathrm{Co}}$.

Proposition 4. The following diagram commutes.

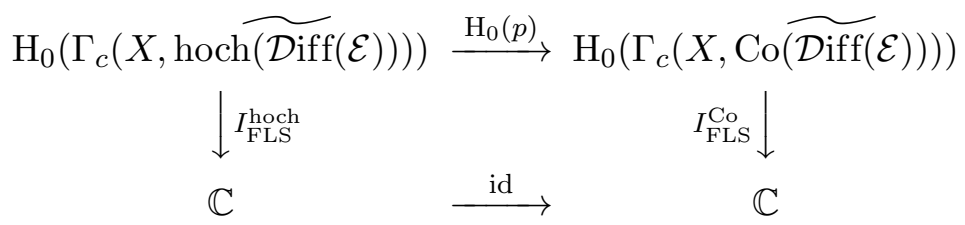

Note that if $\beta$ is a nontrivial 0 -cycle of $\left.\Gamma_{c}(X, \operatorname{hoch} \widetilde{(\mathcal{D i f f}}(\mathcal{E}))\right)$, the above proposition and Theorem 4 together imply that $I_{\mathrm{FLS}}^{\mathrm{Co}}(p(\beta))=\frac{1}{(2 \pi i)^{n}} \int_{X}[\beta] \neq 0$. Therefore, $I_{\mathrm{FLS}}^{\mathrm{Co}}$ is a nontrivial linear functional on $\mathrm{H}_{0}\left(\Gamma_{c}(X, \widehat{\operatorname{Co}(\widetilde{\mathcal{D i f f}}(\mathcal{E})))})\right.$.

2.4. Lie algebra homology. We begin this subsection by reminding the reader of a convention that we will follow.

Convention 2. Let $\mathfrak{g}$ be any DG Lie algebra with differential of degree 1 . By the term "complex of Lie chains of $\mathfrak{g}$ ", we shall refer to the total complex of the double complex $\left\{C_{p, q}:=\right.$ $\left.\left(\wedge^{p} \mathfrak{g}\right)_{-q} \mid p \geq 1\right\}$. The horizontal differential is the Chevalley-Eilenberg differential and the vertical differential is that induced by the differential on $\mathfrak{g}$. Note that our coefficients are in the trivial $\mathfrak{g}$-module, and that we are brutally truncating the column of the usual double complex that lies in homological degree 0 . In particular, if $\mathfrak{g}$ is concentrated in degree 0 , we do not consider Lie 0 -chains of $\mathfrak{g}$.

2.4.1. Recall that $\operatorname{Diff}^{\bullet}(\mathcal{E})$ is a sheaf of topological algebras on $X$. Over each open subset $U$ of $X$, the topology on $\Gamma(U, \mathcal{D i f f}(\mathcal{E}))$ is given as in Section 2.1.1. It follows that $\mathfrak{g l}_{r}(\mathcal{D i f f} \bullet(\mathcal{E}))$ is a sheaf of topological Lie algebras on $X$ with the topology on $\Gamma\left(U, \mathfrak{g l}_{r}(\mathcal{D i f f} \bullet(\mathcal{E}))\right)$ induced by the topology on $\Gamma\left(U, \mathcal{D} \operatorname{iff}^{\bullet}(\mathcal{E})\right)$ in the obvious way. Treating $\Gamma\left(U, \mathfrak{g l}_{\infty}^{\text {fin }}\left(\mathcal{D} \operatorname{iff}^{\bullet}(\mathcal{E})\right)\right)$ as the direct limit of the topological vector spaces $\Gamma\left(U, \mathfrak{g l}_{r}(\mathcal{D}\right.$ iff $\left.\bullet(\mathcal{E}))\right)$ makes $\mathfrak{g l}_{\infty}^{\text {fin }}\left(\mathcal{D}\right.$ iff $\left.f^{\bullet}(\mathcal{E})\right)$ a sheaf of topological Lie algebras on $X$. In this case, the complex of "completed" Lie chains $\widehat{\mathrm{C}}_{\bullet}^{\text {lie }}\left(\Gamma\left(U, \mathfrak{g l}_{\infty}^{\mathrm{fin}}\left(\mathcal{D} \operatorname{iff} \mathfrak{E}^{\bullet}(\mathcal{E})\right)\right)\right)$ is obtained by replacing $\wedge^{k} \Gamma\left(U, \mathfrak{g l}_{\infty}^{\mathrm{fin}}(\mathcal{D} \operatorname{iff} \bullet(\mathcal{E}))\right)$ by the image of the idempotent $\frac{1}{k !} \sum_{\sigma \in S_{k}} \operatorname{sgn}(\sigma) \sigma$ acting on $\mathfrak{g l}_{\infty}^{\mathrm{fin}}(\mathbb{C})^{\otimes k} \otimes \Gamma\left(U^{k}, \mathcal{D}\right.$ iff $\left.\left.\boldsymbol{E}^{\bullet}\right)\right)$. Here, $\sigma$ acts on the factors $\mathfrak{g l}_{\infty}^{\mathrm{ffin}}(\mathbb{C})^{\otimes k}$ and $\Gamma\left(U^{k}, \mathcal{D}\right.$ iff $\left.\mathfrak{f}^{\bullet}\left(\mathcal{E}^{\otimes k}\right)\right)$ simultaneously. In an analogous fashion, one can construct the complex $\widehat{\mathrm{C}}_{\bullet}^{\text {lie }}\left(\Gamma\left(U, \mathfrak{g r} \mathfrak{f}_{\infty}^{\mathrm{fin}}(\mathcal{D} \operatorname{iff}(\mathcal{E}))\right)\right)$. Note that $\widehat{\mathrm{C}}_{\bullet}^{\text {lie }}\left(\Gamma\left(U, \mathfrak{g l}_{\infty}^{\mathrm{fin}}(\mathcal{D} \operatorname{iff}(\mathcal{E}))\right)\right)$ is quasi-isomorphic to $\widehat{\mathrm{C}}_{\bullet}^{\text {lie }}\left(\Gamma\left(U, \mathfrak{g l}_{\infty}^{\mathrm{fin}}(\mathcal{D} \operatorname{iff}(\mathcal{E}))\right)\right)$ since the columns of the corresponding double complexes are quasiisomorphic.

Definition 2. We define $\widetilde{\operatorname{Lie}}(\mathcal{D} \operatorname{iff}(\mathcal{E}))$ to be the complex of sheaves associated to the complex of presheaves

$$
U \mapsto \widehat{\mathrm{C}}_{\bullet}^{\text {lie }}\left(\Gamma\left(U, \mathfrak{g r}_{\infty}^{\mathrm{fin}}(\mathcal{D i f f} \bullet(\mathcal{E}))\right)\right)
$$

of $\mathbb{C}$-vector spaces on $X$.

In the proposition below, the subscript ' $c$ ' indicates "compact supports". 


\section{Proposition 5.}

$$
\mathrm{H}_{i}\left(\Gamma_{c}(X, \widetilde{\operatorname{Lie}}(\mathcal{D} \operatorname{iff}(\mathcal{E})))\right) \simeq \mathbb{H}_{c}^{-i}\left(\operatorname{Sym}^{\geq 1}(\underline{\mathbb{C}}[2 n+1] \oplus \underline{\mathbb{C}}[2 n+3] \oplus \cdots)\right) .
$$

Proof. Recall from [12] as well as Section 10.2 of [15] that for any $\mathbb{C}$-algebra $A$, we have an isomorphism

$$
\mathrm{H}_{\geq 1}^{\mathrm{lie}}\left(\mathfrak{g l}_{\infty}^{\mathrm{fin}}(A)\right) \simeq \operatorname{Sym}^{\geq 1}\left(\mathrm{HC}_{\bullet}(A)[1]\right)
$$

Section 10.2 of [15] shows the following facts

(i) The complex $\mathrm{C}_{\bullet}^{\text {lie }}\left(\mathfrak{g l}_{\infty}^{\mathrm{fin}}(A)\right)_{\mathfrak{g l}_{\infty}^{\mathrm{fin}}(\mathbb{C})}$ is a commutative and cocommutative DG-Hopf-algebra.

(ii) One has a quasiisomorphism from the primitive part Prim $\left(\mathrm{C}_{\bullet}^{\text {lie }}\left(\mathfrak{g}_{\infty}^{\mathrm{fin}}(A)\right)_{\mathfrak{g}_{\infty}^{\mathrm{fin}}(\mathbb{C})}\right)$ to the Connes complex $\mathrm{C}_{\bullet}^{\lambda}(A)[1]$.

(iii) The natural map of complexes $\mathrm{C}_{\bullet}^{\text {lie }}\left(\mathfrak{g}_{\infty}^{\mathrm{fin}}(A)\right) \rightarrow \mathrm{C}_{\bullet}^{\text {lie }}\left(\mathfrak{g l}_{\infty}^{\mathrm{ffin}}(A)\right)_{\mathfrak{g l}_{\infty}^{\text {fin }}(\mathbb{C})}$ is a quasi-isomorphism.

It follows from these facts (and the Milnor-Moore and PBW theorems) that one has the quasi-isomorphisms

$$
\begin{aligned}
& \mathrm{C}_{\bullet}^{\text {lie }}\left(\mathfrak{g l}_{\infty}^{\mathrm{fin}}(A)\right) \rightarrow \mathrm{C}_{\bullet}^{\text {lie }}\left(\mathfrak{g l}_{\infty}^{\mathrm{fin}}(A)\right)_{\mathfrak{g r}_{\infty}^{\mathrm{fin}}(\mathbb{C})} \leftarrow \operatorname{Sym}^{\geq 1}\left(\operatorname{Prim}\left(\mathrm{C}_{\bullet}^{\text {lie }}\left(\mathfrak{g}_{\infty}^{\mathrm{fin}}(A)\right)_{\mathfrak{g}_{\infty}^{\mathrm{fin}}(\mathbb{C})}\right)\right), \\
& \operatorname{Sym}^{\geq 1}\left(\operatorname{Prim}\left(\mathrm{C}_{\bullet}^{\text {lie }}\left(\mathfrak{g l}_{\infty}^{\mathrm{fin}}(A)\right)_{\mathfrak{g}_{\infty}^{\mathrm{fin}}(\mathbb{C})}\right)\right) \rightarrow \mathrm{Sym}^{\geq 1}\left(\mathrm{C}_{\bullet}^{\lambda}(A)[1]\right), \\
& \operatorname{Sym}^{\geq 1}(\mathrm{CC} \bullet(A)[1]) \rightarrow \operatorname{Sym}^{\geq 1}\left(\mathrm{C}_{\bullet}^{\lambda}(A)[1]\right) .
\end{aligned}
$$

The reader should recall that we follow Convention 2 when defining the Lie chain complex. The last quasi-isomorphism above is induced by the standard quasi-isomorphism from CC. $(A)$ to $\mathrm{C}_{\bullet}^{\lambda}(A)$. The discussion of Section 10.2 of [15] goes through with the obvious modifications when the algebra $A$ is replaced the sheaf of topological algebras $\mathcal{D} \operatorname{iff}(\mathcal{E})$ and Lie algebra and cyclic chains are completed in the sense mentioned above (see Section 2.3.3 of [4] for a similar assertion in the $\mathrm{C}^{\infty}$ case). Recall from [22] (see also $[23,18]$ ) that the completed cyclic chain complex of $\mathcal{D} \operatorname{iff}(\mathcal{E})$ is quasi-isomorphic to $\underline{\mathbb{C}}[2 n] \oplus \underline{\mathbb{C}}[2 n+2] \oplus \cdots$. It follows that $\widehat{\mathrm{C}}_{\bullet}^{\text {lie }}\left(\mathfrak{g r}_{\infty}^{\mathrm{fin}}(\mathcal{D} \operatorname{iff}(\mathcal{E}))\right)$ is isomorphic to $\operatorname{Sym}^{\geq 1}(\mathbb{C}[2 n+1] \oplus \mathbb{C}[2 n+3] \oplus \cdots)$ in the derived category $\mathrm{D}\left(\operatorname{Sh}_{\mathbb{C}}(X)\right)$ of sheaves of $\mathbb{C}$-vector spaces on $X$. Further note that $\widehat{\mathrm{C}}_{\bullet}^{\text {lie }}\left(\mathfrak{g}_{\infty}^{\mathrm{fin}}(\mathcal{D} \operatorname{iff}(\mathcal{E}))\right)$ is quasi-isomorphic to $\widetilde{\operatorname{Lie}}(\mathcal{D} \operatorname{iff}(\mathcal{E}))\left(\right.$ since $\mathcal{O}_{X}$ is quasi-isomorphic to $\left.\Omega_{X}^{0, \bullet}\right)$. Hence, $\mathbb{H}_{c}^{-i}(\widetilde{\operatorname{Lie}}(\mathcal{D} \operatorname{iff}(\mathcal{E})))$ is isomorphic to $\mathbb{H}_{c}^{-i}\left(\operatorname{Sym}^{\geq 1}(\underline{\mathbb{C}}[2 n+1] \oplus \underline{\mathbb{C}}[2 n+3] \oplus \cdots)\right)$ for all $i$.

As in Section 2.1, one argues that $\widetilde{\operatorname{Lie}}(\mathcal{D} \operatorname{iff}(\mathcal{E}))$ is a complex of flabby sheaves on $X$. Hence, $\mathrm{H}_{i}\left(\Gamma_{c}(X, \widetilde{\operatorname{Lie}}(\mathcal{D} \operatorname{iff}(\mathcal{E})))\right)$ is isomorphic to $\mathbb{H}_{c}^{-i}(\widetilde{\operatorname{Lie}}(\mathcal{D} \operatorname{iff}(\mathcal{E})))$, proving the desired proposition.

Note that $\operatorname{Sym}^{\geq 1}(\underline{\mathbb{C}}[2 n+1] \oplus \underline{\mathbb{C}}[2 n+3] \oplus \cdots)$ is a direct sum of copies of the constant sheaf $\underline{\mathbb{C}}$ concentrated in homological degrees $\geq 2 n+1$. Let $a(n, i)$ denote the number of partitions of $i$ into pairwise distinct summands from the set $\{2 n+1,2 n+3,2 n+5, \ldots\}$. By Proposition 3.2.2 of [14],

Corollary 1. $\mathrm{H}_{i}\left(\Gamma_{c}(X, \widetilde{\operatorname{Lie}}(\mathcal{D} \operatorname{iff}(\mathcal{E})))\right) \simeq \oplus_{j \in \mathbb{Z}} \mathrm{H}_{c}^{j}(X, \mathbb{C})^{a(n, i+j)}$ for all $i \in \mathbb{Z}$. In particular, $\mathrm{H}_{1}\left(\Gamma_{c}(X, \widetilde{\operatorname{Lie}}(\mathcal{D} \operatorname{iff}(\mathcal{E})))\right) \simeq \mathrm{H}_{c}^{2 n}(X, \mathbb{C})$ and $\mathrm{H}_{i}\left(\Gamma_{c}(X, \widetilde{\operatorname{Lie}}(\mathcal{D i f f}(\mathcal{E})))\right) \simeq 0 \forall i \leq 0$.

2.4.2. For any algebra $A$, we have a map of complexes

$$
L: \quad \mathrm{C}_{\bullet}^{\mathrm{lie}}\left(\mathfrak{g l}_{\infty}^{\mathrm{fin}}(A)\right)[1] \rightarrow \mathrm{C}_{\bullet}^{\lambda}(A),
$$

where the right hand side is the Connes complex of $A$ (the quotient of the Hochschild chain complex of $A$ by the image of $1-t$ ). To be explicit, a Lie $k+1$ chain $M_{0} \wedge \cdots \wedge M_{k}$ maps to 
the $k$-chain $\sum_{\sigma \in S_{k}} M_{0} \otimes M_{\sigma(1)} \otimes \cdots \otimes M_{\sigma(k)}$ of $\mathrm{C}_{\bullet}^{\lambda}\left(M_{N}(A)\right)$ for $N$ sufficiently large. One then applies the generalized trace map to obtain a $k$ chain in $\mathrm{C}_{\bullet}^{\lambda}(A)$.

It is easily verified that the map $L$ extends by continuity to a map of complexes from $\widehat{\mathrm{C}}_{\bullet}^{\text {lie }}\left(\Gamma\left(U, \mathfrak{g l}_{\infty}^{\mathrm{fin}}\left(\mathcal{D}\right.\right.\right.$ iff $\left.\left.\left.\mathfrak{E}^{\bullet}(\mathcal{E})\right)\right)\right)[1]$ to the completed Connes complex for $\Gamma\left(U, \mathcal{D}\right.$ iff $\left.\mathfrak{f}^{\bullet}(\mathcal{E})\right)$. By sheafification followed by taking global sections, it further yields a map of complexes from

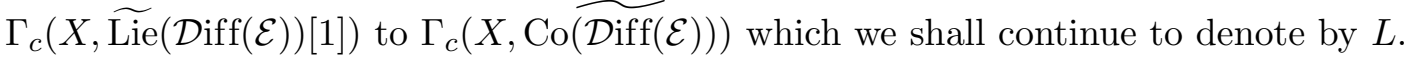

Proposition 6. L induces a nonzero map from

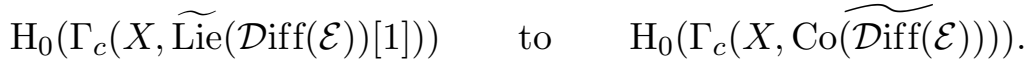

Proof. Recall from [15] that for any algebra $A, L$ induces an isomorphism between the primitive part of $\mathrm{H}_{\bullet}^{\text {lie }}\left(\mathfrak{g l}_{\infty}^{\mathrm{fin}}(A)\right)$ and $\mathrm{HC}_{\bullet-1}(A)$. For the topological algebra $\Gamma\left(U, \mathcal{D}\right.$ iff $\left.\boldsymbol{E}^{\bullet}(\mathcal{E})\right)$ on a sufficiently small open subset $U$ of $X$, it induces an isomorphism between the primitive part of $\mathrm{H}_{\bullet}\left(\widehat{\mathrm{C}}_{\bullet}^{\text {lie }}\left(\Gamma\left(U, \mathfrak{g l}_{\infty}^{\mathrm{fin}}(\mathcal{D} \operatorname{iff} \bullet(\mathcal{E}))\right)\right)\right)$ and the completed cyclic homology of $\Gamma(U, \mathcal{D} \operatorname{iff}(\mathcal{E}))$. In particular, it induces an isomorphism between

$$
\mathrm{H}_{2 n}\left(\widehat{\mathrm{C}}_{\bullet}^{\text {lie }}\left(\Gamma\left(U, \mathfrak{g f}_{\infty}^{\mathrm{fin}}(\mathcal{D i f f} \bullet(\mathcal{E}))\right)\right)[1]\right) \quad \text { and } \quad \mathrm{H}_{2 n}\left(\widehat{\mathrm{C}}_{\bullet}^{\lambda}\left(\Gamma\left(U, \mathfrak{g l}_{\infty}^{\mathrm{fin}}\left(\mathcal{D} \operatorname{iff} \mathbf{f}^{\bullet}(\mathcal{E})\right)\right)\right)\right)
$$

both of which are isomorphic to $\mathbb{C}$. The desired proposition follows from this.

It follows from Propositions 4 and 6 that the map $I_{\mathrm{FLS}}^{\mathrm{Co}} \circ L$ induces a nonzero linear functional on $\mathrm{H}_{0}\left(\Gamma_{c}(X, \widetilde{\operatorname{Lie}}(\mathcal{D} \operatorname{iff}(\mathcal{E}))[1])\right)$. We will denote this linear functional by $I_{\mathrm{FLS}}^{\text {lie }}$ at the level of chains as well as on homology. Note that if $\alpha$ is a 0 -chain in $\Gamma_{c}(X, \widetilde{\operatorname{Lie}}(\mathcal{D} \operatorname{iff}(\mathcal{E}))[1])$, the component of $L(\alpha)$ in $\operatorname{Diff}^{0}(\mathcal{E})$ is $\operatorname{Tr}\left(\alpha_{0}\right)$ where $\alpha_{0}$ is the component of $\alpha$ in $\mathfrak{g f}_{\infty}^{\text {fin }}\left(\operatorname{Diff}^{0}(\mathcal{E})\right)$ and $\operatorname{Tr}$ is the usual matrix trace. Therefore,

$$
I_{\mathrm{FLS}}^{\mathrm{lie}}(\alpha)=\lim _{t \rightarrow \infty} \operatorname{str}\left(\operatorname{Tr}\left(\alpha_{0}\right) e^{-t \Delta_{\mathcal{E}}}\right) .
$$

Let $\mathcal{F}$ be another holomorphic vector bundle on $X$. There is a natural map $\iota: \mathcal{D i f f}^{\bullet}(\mathcal{E}) \rightarrow$ $\mathcal{D i f f} \bullet(\mathcal{E} \oplus \mathcal{F})$ of DG-algebras on $X$. $\iota$ induces a map

$$
\bar{\iota}: \quad \Gamma_{c}(X, \widetilde{\operatorname{Lie}}(\mathcal{D} \operatorname{iff}(\mathcal{E}))[1]) \rightarrow \Gamma_{c}(X, \widetilde{\operatorname{Lie}}(\mathcal{D} \operatorname{iff}(\mathcal{E} \oplus \mathcal{F}))[1]) .
$$

Proposition 7. The following diagram commutes

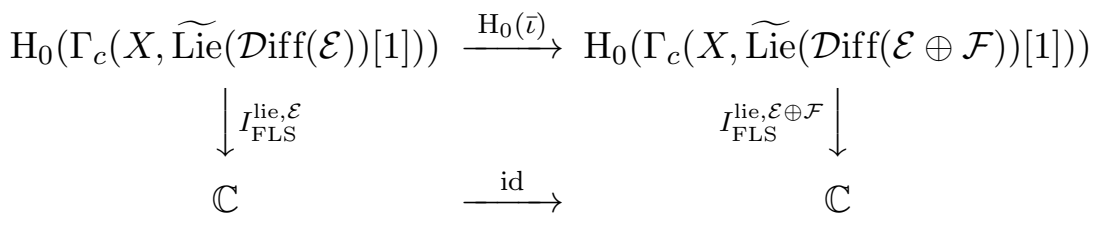

Proof. Let $\alpha$ be a 0 -chain in $\Gamma_{c}(X, \widetilde{\operatorname{Lie}}(\mathcal{D} \operatorname{iff}(\mathcal{E}))[1])$. Clearly, $\operatorname{Tr}\left(\bar{\iota}(\alpha)_{0}\right)=\iota\left(\operatorname{Tr}\left(\alpha_{0}\right)\right)$. It then follows immediately that

$$
\lim _{t \rightarrow \infty} \operatorname{str}\left(\operatorname{Tr}\left(\bar{\iota}\left(\alpha_{0}\right)\right) e^{-t \Delta_{\mathcal{E} \oplus \mathcal{F}}}\right)=\lim _{t \rightarrow \infty} \operatorname{str}\left(\operatorname{Tr}\left(\alpha_{0}\right) e^{-t \Delta_{\mathcal{E}}}\right)
$$

provided the Hermitian metric on $\mathcal{E} \oplus \mathcal{F}$ is chosen by retaining that on $\mathcal{E}$ and choosing an arbitrary Hermitian metric on $\mathcal{F}$. Part 2 of Theorem 4 however, implies that on homology, $I_{\mathrm{FLS}}^{\mathrm{lie}, \mathcal{E} \oplus \mathcal{F}}$ is independent of the choice of Hermitian metric on $\mathcal{E} \oplus \mathcal{F}$. This proves the desired proposition. 


\section{Differential forms computing the Lefschetz number}

3.1. Fedosov differentials. The material in this subsection is a by and large a rehash of material from [10] and [20]. We extend a Gelfand-Fuks type construction from [10] in this section. This construction is a version of a construction that was originally done in [13] by I.M. Gel'fand and D.B. Fuks for Lie cocycles with trivial coefficients for the Lie algebra of smooth vector fields on a smooth manifold. We begin by recalling the construction from [10].

3.1.1. Let $r$ be the rank of $\mathcal{E}$. Let $J_{p} \mathcal{E}$ denote the bundle of $p$-jets of local trivializations of $\mathcal{E}$. In particular, $J_{1} \mathcal{E}$ is the extended frame bundle whose fiber over each $x \in X$ consists of the set of all pairs comprising a basis of $T_{X, x}^{1,0}$ and a basis of $\mathcal{E}_{x}$. The group $G:=G L(n, \mathbb{C}) \times G L(r, \mathbb{C})$ acts on the right on $J_{p} \mathcal{E}$ for each $p$. More specifically, given a local isomorphism of bundles $\mathbb{C}^{n} \times \mathbb{C}^{r} \rightarrow \mathcal{E}, G L(n, \mathbb{C})$ acts by linear coordinate transformations on $\mathbb{C}^{n}$ and $G L(r, \mathbb{C})$ acts by linear transformations on $\mathbb{C}^{r}$. This makes $J_{1} \mathcal{E}$ a principal $G$-bundle. Let $J_{\infty} \mathcal{E}$ denote $\lim J_{p} \mathcal{E}$. Since the natural submersions $J_{p+1} \mathcal{E} \rightarrow J_{p} \mathcal{E}$ are $G$-equivariant, the natural map $J_{\infty} \mathcal{E} \rightarrow J_{1} \mathcal{E}$ descends to a map $J_{\infty} \mathcal{E} / G \rightarrow J_{1} \mathcal{E} / G=X$. Since the fibres of this map are contractible (see [10]), there exists a smooth section $\varphi$ of $J_{\infty} \mathcal{E} / G$ over $X$. This is equivalent to a $G$-equivariant section of $J_{\infty} \mathcal{E}$ over $J_{1} \mathcal{E}$. This section is unique upto smooth homotopy.

3.1.2. Let $\mathcal{O}_{n}$ denote $\mathbb{C}\left[\left[y_{1}, \ldots, y_{n}\right]\right]$. Let $W_{n}$ denote the Lie algebra of formal holomorphic vector fields (i.e., those of the form $\sum_{i=1}^{n} f_{i}\left(y_{1}, \ldots, y_{n}\right) \partial_{i}$ with $f_{i} \in \mathcal{O}_{n}$ and $\left.\partial_{i}:=\frac{\partial}{\partial y_{i}}\right)$. Let $W_{n, r}$ denote the Lie algebra $W_{n} \ltimes \mathfrak{g l}_{r}\left(\mathcal{O}_{n}\right)$. This semidirect product comes from the action of $W_{n}$ on $\mathfrak{g l}\left(\mathcal{O}_{n}\right)$ by derivations. We recall from [10] that there is a map of Lie algebras from $W_{n, r}$ to the Lie algebra of holomorphic vector fields on $J_{\infty} \mathcal{E}$ which yields an isomorphism $W_{n, r} \rightarrow T_{\phi}^{1,0} J_{\infty} \mathcal{E}$ for each $\phi \in J_{\infty} \mathcal{E}$. This is equivalent to a $W_{n, r}$-valued holomorphic 1-form $\Omega$ on $J_{\infty} \mathcal{E}$ satisfying the Maurer-Cartan equation

$$
d \Omega+\frac{1}{2}[\Omega, \Omega]=0
$$

Since there is a natural map of Lie algebras $W_{n, r} \rightarrow \mathrm{M}_{r}\left(\operatorname{Diff}_{n}\right), \Omega$ may be viewed as a MaurerCartan form with values in $\mathrm{M}_{r}\left(\operatorname{Diff}_{n}\right)$.

We clarify that for each $\phi=\left(\phi_{p}\right)$ a point in $J_{\infty} \mathcal{E}, T_{\phi}^{1,0} J_{\infty} \mathcal{E}:=\lim _{\longleftarrow} T_{\phi_{p}}^{1,0} J_{p} \mathcal{E}$ and $\Omega_{\phi}^{1,0} J_{\infty} \mathcal{E}:=$ $\underset{\lim }{\longrightarrow} \Omega_{\phi_{p}}^{1,0} J_{p} \mathcal{E}$, etc. This is compatible with the definition of $J_{\infty} \mathcal{E}$ as $\varliminf_{\longleftarrow} J_{p} \mathcal{E}$. This enables us to make sense of tangent and cotangent spaces of the "infinite dimensional complex manifold" $J_{\infty} \mathcal{E}$, and hence, make sense of vector fields and differential forms on $J_{\infty} \mathcal{E}$.

3.1.3. Consider the bundle $\mathcal{B}_{\mathcal{E}}:=J_{1} \mathcal{E} \times{ }_{G} \mathrm{M}_{r}\left(\right.$ Diff $\left._{n}\right)$ of algebras with fiberwise product. Any trivialization of $J_{1} \mathcal{E}$ over an open subset $U$ of $X$ yields an isomorphism of algebras

$$
\mathrm{C}^{\infty}\left(U, \mathcal{B}_{\mathcal{E}}\right) \simeq \mathrm{C}^{\infty}\left(U, \mathrm{M}_{r}\left(\operatorname{Diff}_{n}\right)\right)
$$

More generally, a trivialization of $J_{1} \mathcal{E}$ over $U$ yields an isomorphism of graded algebras

$$
\left.\Omega^{\bullet}\left(U, \mathcal{B}_{\mathcal{E}}\right)\right) \rightarrow \Omega^{\bullet}\left(U, \mathrm{M}_{r}\left(\operatorname{Diff}_{n}\right)\right) .
$$

The $G$ equivariant section $\varphi: J_{1} \mathcal{E} \rightarrow J_{\infty} \mathcal{E}$ allows us to pull back $\Omega$. This gives us a one form $\omega:=$ $\varphi^{*} \Omega \in \Omega^{1}\left(J_{1} \mathcal{E}, \mathrm{M}_{r}\left(\operatorname{Diff}_{n}\right)\right)^{G}$. This in turn yields a flat connection $D$ on the bundle of algebras $\mathcal{B}_{\mathcal{E}}$. Let us be explicit here. Given a trivialization of $J_{1} \mathcal{E}$ over $U, \omega$ descends to a $\mathrm{M}_{r}\left(\operatorname{Diff}_{n}\right)$ valued one form $\omega_{U}$ on $U$ satisfying the Maurer-Cartan equation. The isomorphism (5) identified the connection $D$ with the "twisted" de Rham differential $d+\left[\omega_{U},-\right]$. More generally, the DGalgebra $\left(\Omega^{\bullet}\left(U, \mathcal{B}_{\mathcal{E}}\right), D\right)$ is identified with the DG-algebra $\left(\Omega^{\bullet}\left(U, \mathrm{M}_{r}\left(\operatorname{Diff}_{n}\right)\right), d+\left[\omega_{U},-\right]\right)$. Over any open subset $U$ of $X$, the degree 0 sections $\hat{\mathcal{D}}$ of $\Omega^{\bullet}\left(\mathcal{B}_{\mathcal{E}}\right)$ satisfying

$$
D \hat{\mathcal{D}}=0
$$


are in one-to-one correspondence with holomorphic differential operators on $\mathcal{E}$ over $U$. The differential $D$ is a Fedosov differential on the sheaf $\Omega^{\bullet}\left(\mathcal{B}_{\mathcal{E}}\right)$ of DG-algebras on $X$.

3.1.4. Fedosov differentials have earlier been constructed in a holomorphic setting in [16], followed by [6] and more recently, [7]. Construction of a Fedosov differential $D$ on $\Omega^{\bullet}\left(\mathcal{B}_{\mathcal{E}}\right)$ has been explained in [7] (see also Dolgushev, [8]) in the case when $\mathcal{E}=\mathcal{O}_{X}$. This goes through with minor modifications for the case when $\mathcal{E}$ is arbitrary. This is a more "careful" construction of a Fedosov differential: it has the following special property (Part 2 of Theorem 10.5 of [7]: note that we do not need Part 1 of this result).

Theorem 5. There is a map of sheaves of DG-algebras

$$
\operatorname{Diff}^{\bullet}(\mathcal{E}) \rightarrow\left(\Omega^{\bullet}\left(\mathcal{B}_{\mathcal{E}}\right), D\right) .
$$

Given that the construction of the Fedosov differential $D$ from [7] is a priori different from that of [10], one would like to know whether the Fedosov differential $D$ from [7] can also be obtained via the construction from [10] outlined earlier. The reason why we need this relationship will become clear in later sections. The following paragraphs give a partial affirmative answer to this question that suffices for us. To be precise, the next paragraph shows that there exists a $G$ equivariant section $\varphi: J_{1} \mathcal{E} \rightarrow J_{\infty} \mathcal{E}$ associated with any Fedosov differential on $\Omega^{\bullet}\left(\mathcal{B}_{\mathcal{E}}\right)$. Further, Proposition 8 in Section 3.1.5 shows that for every $\mathcal{D} \in \Gamma(U, \mathcal{D} \operatorname{iff}(\mathcal{E}))$, the section of $\mathcal{B}_{\mathcal{E}}$ over $U$ that $\mathcal{D}$ yields via $\varphi$ (via the construction in Section 3.1.3) coincides with the flat (with respect to $D$ ) section of $\mathcal{B}_{\mathcal{E}}$ corresponding to $\mathcal{D}$. We also point out that on any open subset $U$ of $X$ on which $J_{1} \mathcal{E}$ is trivial, any trivialization of $J_{1} \mathcal{E}$ over $U$ identifies the DG-algebra $\left(\Omega^{\bullet}\left(U, \mathcal{B}_{\mathcal{E}}\right), D\right)$ with the DG-algebra $\left(\Omega^{\bullet}\left(U, \mathrm{M}_{r}\left(\operatorname{Diff}_{n}\right)\right), d+\left[\omega_{U},-\right]\right)$ for some $\mathrm{M}_{r}\left(\operatorname{Diff}_{n}\right)$-valued Maurer-Cartan form even in the construction of [7].

3.1.5. The argument here follows Section 4.3 of [11]. Consider any point $(x, F)$ of $J_{1} \mathcal{E}$ ( $F$ being an extended frame over $x$ ). For any holomorphic differential operator $\mathcal{D}$ on $\mathcal{E}$ over a neighborhood of $x$, we have a unique section $\hat{\mathcal{D}}$ of $\Omega\left(\mathcal{B}_{\mathcal{E}}\right)$ satisfying $D \hat{\mathcal{D}}=0$. The frame $F$ identifies $\hat{\mathcal{D}}$ with an element of $\mathrm{M}_{r}\left(\right.$ Diff $\left._{n}\right)$. This yields an isomorphism of algebras

$$
\Theta: \operatorname{Jets}_{x} \mathcal{D} \operatorname{iff}(\mathcal{E}) \rightarrow \mathrm{M}_{r}\left(\operatorname{Diff}_{n}\right) .
$$

Moreover, since the above isomorphism preserves the order of the differential operator, we obtain an isomorphism

$$
\theta: \quad \operatorname{Jets}_{x} \operatorname{End}(\mathcal{E}) \rightarrow \mathrm{M}_{r}\left(\mathcal{O}_{n}\right)
$$

This corresponds to an element in the fibre of $J_{\infty} \mathcal{E}$ over $x$. We therefore, obtain a section $\varphi$ : $J_{1} \mathcal{E} \rightarrow J_{\infty} \mathcal{E}$. It is easy to verify that this section is $G$-equivariant. Note that the isomorphism $\theta$ induces an isomorphism $\tilde{\theta}$ from $\operatorname{Jets}_{x} \mathcal{D} \operatorname{iff}(\mathcal{E})$ to $\mathrm{M}_{r}\left(\operatorname{Diff}_{n}\right)$.

Proposition 8. $\tilde{\theta}$ coincides with $\Theta$.

Proof. Note that $\mathrm{M}_{r}\left(\mathcal{O}_{n}\right)$ and Id $\otimes W_{n}$ generate $\mathrm{M}_{r}\left(\operatorname{Diff}_{n}\right)$ as an algebra. It therefore suffices to check that $\tilde{\theta}^{-1}$ and $\Theta^{-1}$ coincide on $\mathrm{M}_{r}\left(\mathcal{O}_{n}\right)$ and $\mathrm{Id} \otimes W_{n}$. Observe that the image of $W_{n, r}:=$ $W_{n} \ltimes \mathfrak{g l}_{r}\left(\mathcal{O}_{n}\right)$ in $\mathrm{M}_{r}\left(\operatorname{Diff}_{n}\right)$ is precisely the space of all elements of the form $X=D+f$ for some $D \in \mathrm{Id} \otimes W_{n}$ and some $f \in \mathrm{M}_{r}\left(\mathcal{O}_{n}\right)$. We shall continue to denote this image by $W_{n, r}$.

Claim. Every derivation of $\mathrm{M}_{r}\left(\mathcal{O}_{n}\right)$ is of the form $[X,-]$ where $X \in W_{n, r}$. Further, if $X \in \mathrm{M}_{r}\left(\operatorname{Diff}_{n}\right)$ and $[X,-]$ is a derivation of $\mathrm{M}_{r}\left(\mathcal{O}_{n}\right)$, then $X \in W_{n, r}$.

We first prove the proposition assuming our claim. Indeed, $\Theta^{-1}$ restricted to $\mathrm{M}_{r}\left(\mathcal{O}_{n}\right)$ coincides with $\tilde{\theta}^{-1}$ restricted to $\mathrm{M}_{r}\left(\mathcal{O}_{n}\right)$ (both of which coincide with $\left.\theta^{-1}\right)$. For any $X \in W_{n, r}$ and for any $g \in \mathrm{M}_{r}\left(\mathcal{O}_{n}\right),\left[\tilde{\theta}^{-1}(X), \theta^{-1}(g)\right]=\tilde{\theta}^{-1}([X, g])=\theta^{-1}([X, g])=\Theta^{-1}([X, g])=\left[\Theta^{-1} X, \theta^{-1} g\right]$. It follows that for any $X \in W_{n, r}, \tilde{\theta}^{-1}(X)-\Theta^{-1}(X)$ commutes with all elements of $\operatorname{Jets}_{x} \operatorname{End}(\mathcal{E})$. 
In other words, $\tilde{\theta}^{-1}(X)-\Theta^{-1}(X)$ must be an element of $\theta^{-1}\left(\mathrm{id} \otimes \mathcal{O}_{n}\right)$ for all $X \in W_{n, r}$. In particular, if $X \in \mathrm{id} \otimes W_{n}$, then $\tilde{\theta}^{-1}(X)=\Theta^{-1}(X)+\theta^{-1}(g)$ for some $g \in \mathrm{id} \otimes \mathcal{O}_{n}$. In this case,

$$
\begin{aligned}
\tilde{\theta}^{-1}\left(X^{2}\right) & =\Theta^{-1}\left(X^{2}\right)+\Theta^{-1}(X) \cdot \theta^{-1}(g)+\theta^{-1}(g) \cdot \Theta^{-1}(X)+\theta^{-1}\left(g^{2}\right) \\
& =\Theta^{-1}\left(X^{2}\right)+\Theta^{-1}(2 g X)+\theta^{-1}\left([X, g]+g^{2}\right) .
\end{aligned}
$$

It follows that $\tilde{\theta}^{-1}\left(X^{2}\right)-\Theta^{-1}\left(X^{2}\right)$ is in $\theta^{-1}\left(\mathrm{id} \otimes \mathcal{O}_{n}\right)$ only if $g=0$. This shows that $\tilde{\theta}^{-1}$ and $\Theta^{-1}$ coincide on id $\otimes W_{n}$ as well.

Our claim remains to be proven. Recall (from [15] for instance) that for any algebra $A$,

$$
\mathrm{HH}^{1}(A) \simeq \frac{\operatorname{Der}(A, A)}{\text { Inner derivations }} .
$$

In our case, the composite map

$$
W_{n} \rightarrow \mathrm{HH}^{1}\left(\mathcal{O}_{n}, \mathcal{O}_{n}\right) \rightarrow \mathrm{HH}^{1}\left(\mathrm{M}_{r}\left(\mathcal{O}_{n}\right), \mathrm{M}_{r}\left(\mathcal{O}_{n}\right)\right)
$$

is an isomorphism. The first arrow above is the Hochschild-Kostant-Rosenberg map. The second arrow above is the cotrace. It follows that for any derivation $\phi$ of $\mathrm{M}_{r}\left(\operatorname{Diff}_{n}\right)$, there is an element $v$ of $W_{n}$ such that the image of $v$ under the above composite map coincides with that of $\phi$ in $\mathrm{HH}^{1}\left(\mathrm{M}_{r}\left(\mathcal{O}_{n}\right), \mathrm{M}_{r}\left(\mathcal{O}_{n}\right)\right)$. Consider the derivation $\bar{v}:=[\mathrm{id} \otimes v,-]$ of $\mathrm{M}_{r}\left(\mathcal{O}_{n}\right)$. It is easy to verify that the image of $\phi-\bar{v}$ in $\operatorname{HH}^{1}\left(\mathrm{M}_{r}\left(\mathcal{O}_{n}\right), \mathrm{M}_{r}\left(\mathcal{O}_{n}\right)\right)$ is zero. Hence, $\phi-\bar{v}$ is an inner derivation. In other words, as derivations on $\mathrm{M}_{r}\left(\mathcal{O}_{n}\right), \phi=[\mathrm{id} \otimes v+f,-]$ for some $v \in W_{n}$ and some $f \in \mathrm{M}_{r}\left(\mathcal{O}_{n}\right)$. This proves the first part of our claim. The second part of our claim is then easy: if $[X,-]$ is a derivation of $\mathrm{M}_{r}\left(\mathcal{O}_{n}\right)$, then $[X,-]=[Y,-]$ as derivations on $\mathrm{M}_{r}\left(\mathcal{O}_{n}\right)$ for some $Y \in W_{n, r}$. Hence, $X-Y$ commutes with every element in $\mathrm{M}_{r}\left(\mathcal{O}_{n}\right)$. This shows that $X-Y$ is an element of id $\otimes \mathcal{O}_{n}$.

3.2. A Gel'fand-Fuks type construction. We now recall and extend a construction from [10].

3.2.1. Let $A$ be any DG-algebra. Let $\omega \in A^{1}$ be a Maurer-Cartan element (i.e., an satisfying $\left.d \omega+\omega^{2}=0\right)$. Let $A_{\omega}$ denote the DGA whose underlying graded algebra is $A$ but whose differential is $d+[\omega,-]$. Let $\mathrm{C} \cdot(A)$ denote the complex of normalized Hochschild chains of $A$. Let $(\omega)^{k}$ denote the normalized Hochschild 0 -chain $(1, \omega, \ldots, \omega)(k$ copies of $\omega)$. Denote the set of $(p, q)$-shuffles by $\mathrm{Sh}_{p . q}$. There is a shuffle product

$$
\begin{aligned}
& \times: \quad A \otimes A^{\otimes p} \otimes A \otimes A^{\otimes q} \rightarrow A \otimes A^{\otimes p+q}, \\
& \left(a_{0}, a_{1}, \ldots, a_{p}\right) \otimes\left(b_{0}, a_{p+1}, \ldots, a_{p+q}\right) \\
& \quad \mapsto \sum_{\sigma \in \operatorname{Sh}_{p, q}} \operatorname{sgn}(\sigma)\left(a_{0} b_{0}, a_{\sigma^{-1}(1)}, \ldots, a_{\sigma^{-1}(p)}, a_{\sigma^{-1}(p+1)}, \ldots, a_{\sigma^{-1}(p+q)}\right) .
\end{aligned}
$$

Note that $\mathrm{C}_{\bullet}(A)=\oplus_{k \geq 1} A \otimes A^{\otimes k}[k-1]$ as graded vector spaces. The differential on $\mathrm{C}_{\bullet}(A)$ extends naturally to a differential on $\mathrm{C}_{\bullet}^{\Pi}(A):=\prod_{k \geq 1} A \otimes A^{\otimes k}[k-1]$ making the natural map of graded vector spaces a map of complexes. One has the following proposition due to Engeli and Felder [10]. Though [10] used normalized Hochschild chains, their proof goes through in the current context as well.

Proposition 9. The map

$$
\mathrm{C}_{\bullet}^{\Pi}\left(A_{\omega}\right) \rightarrow \mathrm{C}_{\bullet}^{\Pi}(A), \quad x \mapsto x \times \sum_{k}(-1)^{k}(\omega)^{k}
$$

is a term by term isomorphism of complexes. 
3.2.2. Recall that $G L(n, \mathbb{C})$ acts on a formal neighborhood of the origin by linear coordinate changes. This induces an action of $G L(n, \mathbb{C})$ on $\operatorname{Diff}_{n}$. The derivative of this action embeds the Lie algebra $\mathfrak{g l}_{n}$ in $\operatorname{Diff}_{n}$ as the Lie subalgebra of operators of the form $\sum_{j, k} a_{j, k} y_{k} \partial_{j}$. We say that the normalized cocycle $\alpha \in \mathrm{C}^{p}\left(\operatorname{Diff}_{n}\right)$ is $G L(n, \mathbb{C})$-basic if $\alpha$ is $G L(n, \mathbb{C})$-invariant and if $\sum_{i=1}^{p}(-1)^{i+1} \alpha\left(a_{0}, \ldots, a_{i-1}, a, a_{i+1}, \ldots, a_{p}\right)=0$ for any $a \in \mathfrak{g l}_{n}$ and any $a_{0}, \ldots, a_{p} \in \operatorname{Diff}_{n}$. More generally, $G:=G L(n, \mathbb{C}) \times G L(r, \mathbb{C})$ acts on $\mathrm{M}_{r}\left(\operatorname{Diff}_{n}\right)$. The action of $G L(n, \mathbb{C})$ on $\mathrm{M}_{r}\left(\operatorname{Diff}_{n}\right)$ is induced by the action of $G L(n, \mathbb{C})$ on $\operatorname{Diff}_{n}$ and $G L(r, \mathbb{C})$ acts on $\mathrm{M}_{r}\left(\operatorname{Diff}_{n}\right)$ by conjugation. The derivative of this action embeds $\mathfrak{g}:=\mathfrak{g l}_{n} \oplus \mathfrak{g l}_{r}$ as a Lie subalgebra of $\mathrm{M}_{r}\left(\operatorname{Diff}_{n}\right)$ of elements of the form $\sum_{j, k} a_{j, k} z_{k} \partial_{j} \otimes \mathrm{id}_{r \times r}+B$ where $B \in \mathrm{M}_{r}(\mathbb{C})$. Again, a cocycle $\alpha$ in $\mathrm{C}^{p}\left(\mathrm{M}_{r}\left(\operatorname{Diff}_{n}\right)\right)$ is said to be $G$ basic if $\alpha$ is $G$-invariant and $\sum_{i=1}^{p}(-1)^{i+1} \alpha\left(a_{0}, \ldots, a_{i-1}, a, a_{i+1}, \ldots, a_{p}\right)=0$ for any $a \in \mathfrak{g}$ and any $a_{0}, \ldots, a_{p} \in \mathrm{M}_{r}\left(\operatorname{Diff}_{n}\right)$. The following proposition is immediate from equation (1).

Proposition 10. If $\psi_{2 n}$ is $G L(n, \mathbb{C})$-basic then $\psi_{2 n}^{r}$ is $G$-basic.

We also recall that a cocycle $\alpha \in \mathrm{C}^{p}\left(\mathrm{M}_{r}\left(\operatorname{Diff}_{n}\right)\right)$ is said to be continuous if it depends only on finitely many Taylor coefficients of its arguments.

3.2.3. Let $\psi_{2 n}^{r}$ be a continuous, $G$-basic Hochschild $2 n$-cocycle of $\mathrm{M}_{r}\left(\operatorname{Diff}_{n}\right)$. Evaluation at $\psi_{2 n}^{r}$ gives a map of graded vector spaces

$$
\begin{aligned}
& \mathrm{C}_{\bullet}^{\Pi}\left(\Omega^{\bullet}\left(U, M_{r}\left(\operatorname{Diff}_{n}\right)\right), d\right) \rightarrow \Omega^{2 n-\bullet}(U), \\
& \left(\omega_{0} a_{0}, \ldots, \omega_{p} a_{p}\right) \mapsto \psi_{2 n}^{r}\left(a_{0}, \ldots, a_{p}\right) \omega_{1} \wedge \cdots \wedge \omega_{p}
\end{aligned}
$$

for all $a_{1}, \ldots, a_{p} \in \mathrm{M}_{r}\left(\operatorname{Diff}_{n}\right), \omega_{1}, \ldots, \omega_{p} \in \Omega^{\bullet}(U)$.

By convention, $\psi_{2 n}^{r}\left(a_{0}, \ldots, a_{p}\right)=0$ if $p \neq 2 n$.

Proposition 11. The map (6) is a map of complexes (with $\Omega^{2 n-p}(U)$ equipped with the differential $\left.(-1)^{p} d_{\mathrm{DR}}\right)$.

Proof. Note that any Hochschild $p$-chain $\alpha$ of $A:=\left(\Omega^{\bullet}\left(U, \mathrm{M}_{r}\left(\operatorname{Diff}_{n}\right)\right), d\right)$ can be expressed as a sum of terms $\sum_{i} \alpha_{i}$ where $\alpha_{i}$ has de Rham degree $i$ and is an element of $A \otimes A^{p+i}$. By definition, $\psi_{2 n}^{r}(\alpha)=\psi_{2 n}^{r}\left(\alpha_{2 n-p}\right)$. Let $\delta$ denote the differential on the Hochschild chain complex of $A$. Then, $(\delta \alpha)_{2 n-p}=(-1)^{p} d_{\mathrm{DR}} \alpha_{2 n-p}+d_{\mathrm{hoch}} \alpha_{2 n-p+1}$. Since $\psi_{2 n}^{r}$ is a cocycle, $\psi_{2 n}^{r}\left(d_{\mathrm{hoch}} \alpha_{2 n-p+1}\right)=0$. The desired proposition is now immediate.

Observe that there is a natural map of complexes $\mathrm{C}_{\bullet}\left(\Omega^{\bullet}\left(U, \mathcal{B}_{\mathcal{E}}\right), D\right) \rightarrow \mathrm{C}_{\bullet}^{\Pi}\left(\Omega^{\bullet}\left(U, \mathcal{B}_{\mathcal{E}}\right), D\right)$. Also, the graded involution on $\Omega^{\bullet}(U)$ acting on $\Omega^{p}(U)$ by multiplication by $(-1)^{\left\lfloor\frac{p}{2}\right\rfloor}$ is a map of complexes between $\left(\Omega^{2 n-\bullet}(U),(-1)^{\bullet} d_{\mathrm{DR}}\right)$ and $\Omega^{2 n-\bullet}(U)$. Recall from Section 3.1.4 that a trivialization of $J_{1} \mathcal{E}$ over $U$ identifies $\left(\Omega^{\bullet}\left(U, \mathcal{B}_{\mathcal{E}}\right), D\right)$ with $\Omega_{\omega}^{\bullet}\left(U, \mathrm{M}_{r}\left(\operatorname{Diff}_{n}\right)\right)$ where $\omega$ is a $\mathrm{M}_{r}\left(\right.$ Diff $\left._{n}\right)$-valued 1-form on $U$ satisfying the Maurer-Cartan equation. By Propositions 9 and 11, we have a map of complexes

$$
\mathrm{C} \cdot\left(\Omega^{\bullet}\left(U, \mathcal{B}_{\mathcal{E}}\right), D\right) \rightarrow \Omega^{2 n-\bullet}(U) .
$$

Explicitly, the map (7) maps a chain $\mu \in \mathrm{C}_{p}\left(\Omega^{\bullet}\left(U, \mathcal{B}_{\mathcal{E}}\right), D\right)$ to

$$
(-1)^{\left\lfloor\frac{2 n-p}{2}\right\rfloor} \psi_{2 n}^{r}\left(\hat{\mu} \times \sum_{k}(-1)^{k}(\omega)^{k}\right),
$$

where $\hat{\mu}$ is the image of $\mu$ in $\mathrm{C}_{p}^{\Pi}\left(\Omega_{\omega}^{\bullet}\left(U, \mathrm{M}_{r}\left(\operatorname{Diff}_{n}\right)\right)\right)$.

Proposition 12. The map (7) is independent of the trivialization of $J_{1} \mathcal{E}$ used. 
Proof. A different trivialization of $J_{1} \mathcal{E}$ over $U$ differs from the chosen one by a gauge change $g: U \rightarrow G$. A section $\mu$ of $\Omega^{\bullet}\left(U, \mathcal{B}_{\mathcal{E}}\right)$ transforms into $g . \mu$. The Maurer-Cartan form $\omega$ is replaced by $g . \omega-d g . g^{-1}$. That $\psi_{2 n}^{r}\left(\alpha \times \sum_{k} \pm(\omega)^{k}\right)=\psi_{2 n}^{r}\left(g . \alpha \times \sum_{k} \pm\left(g \cdot \omega-d g \cdot g^{-1}\right)^{k}\right)$ is immediate from the fact that $\psi_{2 n}^{r}$ is $G$-basic. This proves the desired proposition.

It follows that the map (7) gives a map of complexes of presheaves

$$
\left(U \mapsto \mathrm{C}_{\bullet}\left(\Omega^{\bullet}\left(U, \mathcal{B}_{\mathcal{E}}\right), D\right)\right) \rightarrow\left(U \mapsto \Omega^{2 n-\bullet}(U)\right) .
$$

Note that whatever we said so far is true for any Fedosov differential no matter how it was constructed. Of course, the map of complexes of presheaves (8) depends on the Fedosov differential $D$. It follows from Theorem 5 that we obtain a composite map of complexes of presheaves

$$
\left(U \mapsto \mathrm{C}_{\bullet}\left(\mathcal{D i f f}^{\bullet}(\mathcal{E})(U)\right)\right) \rightarrow\left(U \mapsto \mathrm{C}_{\bullet}\left(\Omega^{\bullet}\left(U, \mathcal{B}_{\mathcal{E}}\right), D\right)\right) \rightarrow\left(U \mapsto \Omega^{2 n-\bullet}(U)\right) .
$$

Proposition 13. The composite map (9) extends to a map of complexes of sheaves

$$
\operatorname{hoch(\mathcal {Diff}}(\mathcal{E})) \rightarrow \Omega_{X}^{2 n-\bullet}
$$

Proof. Denote the composite map (9) by $f_{\mathcal{E}}$. For each open subset $U$ of $X, f_{\mathcal{E}}$ yields a map of complexes from C. $\left(\mathcal{D i f f}^{\bullet}(\mathcal{E})(U)\right)$ to $\Omega^{2 n-\bullet}(U)$. As described in Section 2.1.1, $\widehat{\mathrm{C}_{\bullet}}\left(\mathcal{D}\right.$ iff $\left.{ }^{\bullet}(\mathcal{E})(U)\right)$ has the structure of a (graded) locally convex topological vector space. One can verify without difficulty that the subcomplex $\mathrm{C}_{\bullet}\left(\mathcal{D i f f}^{\bullet}(\mathcal{E})(U)\right)$ is dense in $\widehat{\mathrm{C}_{\bullet}}\left(\mathcal{D}\right.$ iff $\left.f^{\bullet}(\mathcal{E})(U)\right)$ and that the differential of $\widehat{\mathrm{C}_{\bullet}}\left(\mathcal{D} \operatorname{iff}^{\bullet}(\mathcal{E})(U)\right)$ is continuous. Similarly, $\Omega^{2 n-\bullet}(U)$ has the structure of a (graded) complete locally convex topological vector space and the de Rham differential is continuous with respect to this topology.

Suppose that $f_{\mathcal{E}}$ is continuous. Then, $f_{\mathcal{E}}$ extends to a map of complexes from $\widehat{\mathrm{C}_{\bullet}}(\mathcal{D}$ iff $\bullet(\mathcal{E})(U))$ to $\Omega^{2 n-\bullet}(U)$ for each $U$. This can be easily see to yield a map of complexes of presheaves

$$
\left(U \mapsto \widehat{\mathrm{C}_{\bullet}}\left(\mathcal{D} \operatorname{iff}^{\bullet}(\mathcal{E})(U)\right)\right) \rightarrow\left(U \mapsto \Omega^{2 n-\bullet}(U)\right)
$$

On sheafification, $f$ yields a map of complexes of sheaves

$$
\operatorname{hoch} \widetilde{(\mathcal{D i f f}}(\mathcal{E})) \rightarrow \Omega_{X}^{2 n-\bullet}
$$

proving the desired proposition. Indeed, continuity of $f_{\mathcal{E}}$ follows from continuity of $\psi_{2 n}^{r}$. Since $\psi_{2 n}^{r}$ is continuous, $C^{l}$-norms of $f\left(a_{0} \otimes \cdots \otimes a_{k}\right)$ over a compact subsets of $U$ are estimated by $C^{l^{\prime}}$-norms of finitely many Taylor coefficients of the images $\hat{a_{i}}$ of the $a_{i}$ in $\Omega^{\bullet}\left(U, \mathcal{B}_{\mathcal{E}}\right)$ over compact subsets of $U$.

The map of complexes of sheaves obtained in Proposition 13 depends on the cocycle $\psi_{2 n}$. We shall denote this map by $f_{\mathcal{E}, \psi_{2 n}}$. Recall that there is a natural map of complexes of sheaves $\widehat{\mathrm{C}} .(\operatorname{Diff}(\mathcal{E})) \rightarrow \operatorname{hoch} \widetilde{(\mathcal{D} \text { iff }}(\mathcal{E}))$. Let $U$ be a subset of $X$ with local holomorphic coordinates $z_{1}, \ldots, z_{n}$ such that $\mathcal{E}$ is trivial over $U$. By Convention 1 , the standard generator for the homology of $\Gamma(U, \widehat{\mathrm{C}} \cdot(\mathcal{D} \operatorname{iff}(\mathcal{E})))$ is a cycle $c_{\mathcal{E}}(U)$ mapping to the normalized Hochschild $2 n$-cycle

$$
\sum_{\sigma \in S_{2 n}} \operatorname{sgn}(\sigma) \sigma\left(\operatorname{Id}_{\mathcal{E}} \otimes 1, \operatorname{Id}_{\mathcal{E}} \otimes z_{1}, \operatorname{Id}_{\mathcal{E}} \otimes \frac{\partial}{\partial z_{1}}, \ldots, \operatorname{Id}_{\mathcal{E}} \otimes z_{n}, \operatorname{Id}_{\mathcal{E}} \otimes \frac{\partial}{\partial z_{n}}\right) .
$$

Let $c_{2 n}^{r}$ denote the normalized Hochschild $2 n$-cycle

$$
\sum_{\sigma \in S_{2 n}} \operatorname{sgn}(\sigma) \sigma\left(\operatorname{Id}_{r \times r} \otimes 1, \operatorname{Id}_{r \times r} \otimes y_{1}, \operatorname{Id}_{r \times r} \otimes \frac{\partial}{\partial y_{1}}, \ldots, \operatorname{Id}_{r \times r} \otimes y_{n}, \operatorname{Id}_{r \times r} \otimes \frac{\partial}{\partial y_{n}}\right)
$$

of $\mathrm{M}_{r}\left(\operatorname{Diff}_{n}\right)$. Note that $\psi_{2 n}^{r}\left(c_{2 n}^{r}\right)$ makes sense since $\psi_{2 n}^{r}$ is $G$-basic. 
Proposition 14. $f_{\mathcal{E}, \psi_{2 n}}\left(c_{\mathcal{E}}(U)\right)=\psi_{2 n}^{r}\left(c_{2 n}^{r}\right)$.

Proof. Note that any element $\mathcal{D}$ of $\mathcal{D} \operatorname{iff}(\mathcal{E})(U)$ gives a holomorphic $\mathrm{M}_{r}\left(\operatorname{Diff}_{n}\right)$-valued function $\tilde{D}$ on $\left.J_{\infty} \mathcal{E}\right|_{U}$, satisfying $d \tilde{\mathcal{D}}+[\Omega, \tilde{\mathcal{D}}]=0$ where $\Omega$ is the Maurer-Cartan form from Section 3.1.2. It follows that there is a map of complexes

$$
\begin{aligned}
\mathrm{C} .(\mathcal{D i f f}(\mathcal{E})(U)) & \rightarrow \mathrm{C}_{\bullet}\left(\left(\Omega^{\bullet, 0}\left(\left.J_{\infty} \mathcal{E}\right|_{U}, M_{r}\left(\operatorname{Diff}_{n}\right)\right), d+[\Omega,-]\right)\right) \\
& \rightarrow \mathrm{C}_{\bullet}^{\Pi}\left(\Omega^{\bullet, 0}\left(\left.J_{\infty} \mathcal{E}\right|_{U}, M_{r}\left(\operatorname{Diff}_{n}\right)\right)\right) .
\end{aligned}
$$

The last arrow is by Proposition 9. Evaluation at $\psi_{2 n}^{r}$ followed by applying the involution multiplying $p$-forms by $(-1)^{\left\lfloor\frac{p}{2}\right\rfloor}$ therefore yields a map of complexes

$$
\text { C. }(\mathcal{D} \operatorname{iff}(\mathcal{E})(U)) \rightarrow \Omega^{2 n-\bullet, 0}\left(\left.J_{\infty} \mathcal{E}\right|_{U}\right) .
$$

Given a section $\varphi: J_{1} \mathcal{E} \rightarrow J_{\infty} \mathcal{E}$ and a trivialization of $J_{1} \mathcal{E}$ over $U$, one has a composite map

$$
\text { C. }(\mathcal{D i f f}(\mathcal{E})(U)) \rightarrow \Omega^{2 n-\bullet, 0}\left(\left.J_{\infty} \mathcal{E}\right|_{U}\right) \rightarrow \Omega^{2 n-\bullet}\left(\left.J_{1} \mathcal{E}\right|_{U}\right) \rightarrow \Omega^{2 n-\bullet}(U) .
$$

The middle arrow above is pullback by the section $\varphi: J_{1} \mathcal{E} \rightarrow J_{\infty} \mathcal{E}$. The rightmost arrow above is pullback by the section of $J_{1} \mathcal{E}$ arising out of the trivialization of $J_{1} \mathcal{E}$ that we have chosen over $U$. Any section $\varphi:\left.\left.J_{1} \mathcal{E}\right|_{U} \rightarrow J_{\infty} \mathcal{E}\right|_{U}$ is unique upto homotopy. It follows from this that the composite map (10) is unique upto homotopy for a fixed trivialization of $J_{1} \mathcal{E}$ over $U$. This fact and the proof of Proposition 12 together imply that the image of $c_{\mathcal{E}}(U)$ under (10) is independent of the precise choice of $\varphi$ and of trivialization of $J_{1} \mathcal{E}$ over $U$. To compute it, we could choose $\varphi$ to be the section taking $\frac{\partial}{\partial z_{i}}$ to the formal derivative $\frac{\partial}{\partial y_{i}}$ and $z_{i}$ to $y_{i}+a_{i}$ at $\left(a_{1}, \ldots, a_{n}\right)$. In this situation, the image of $c_{\mathcal{E}}(U)$ is indeed seen to be $\psi_{2 n}^{r}\left(c_{2 n}^{r}\right)$ in homological degree $2 n$.

For any section $\varphi$ of $\left.J_{\infty} \mathcal{E}\right|_{U}$ over $J_{1} \mathcal{E}$ and for any trivialization of $J_{1} \mathcal{E}$ over $U$, the map (10) takes $\left(\mathcal{D}_{0}, \ldots, \mathcal{D}_{2 n}\right)$ to $\psi_{2 n}^{r}\left(\hat{\mathcal{D}_{0}}, \ldots, \hat{\mathcal{D}_{n}}\right)$ where $\hat{\mathcal{D}}$ is the section of $\Omega^{\bullet}\left(U, \mathrm{M}_{r}\left(\operatorname{Diff}_{n}\right)\right)$ corresponding to $\mathcal{D}$ (satisfying $d \hat{\mathcal{D}}+[\omega, \hat{\mathcal{D}}]=0$ where $\omega$ is as in Section 3.1.3). Let $\varphi$ be a section of $\left.J_{\infty} \mathcal{E}\right|_{U}$ over $J_{1} \mathcal{E}$ associated with the Fedosov differential $D$ on $\Omega^{\bullet}\left(\mathcal{B}_{\mathcal{E}}\right)$ as in Section 3.1.5. Proposition 8 implies that $f_{\mathcal{E}, \psi_{2 n}}$ takes $\left(\mathcal{D}_{0}, \ldots, \mathcal{D}_{2 n}\right)$ to $\psi_{2 n}^{r}\left(\hat{\mathcal{D}_{0}}, \ldots, \hat{\mathcal{D}_{n}}\right)$. Thus, $f_{\mathcal{E}, \psi_{2 n}}\left(c_{\mathcal{E}}(U)\right)=\psi_{2 n}^{r}\left(c_{2 n}^{r}\right)$.

3.3. Properties of $\boldsymbol{f}_{\mathcal{E}, \psi_{2 n}}$. Let $\mathrm{D}^{b}\left(\mathrm{Sh}_{\mathbb{C}}[X]\right)$ denote the bounded derived category of sheaves of $\mathbb{C}$-vector spaces on $X$. Then, the map $f_{\mathcal{E}, \psi_{2 n}}$ of Proposition 13 induces a morphism in $\mathrm{D}^{b}\left(\mathrm{Sh}_{\mathbb{C}}[X]\right)$. Recall that the natural embedding of $\mathbb{C}$ into $\Omega^{\bullet}(U)$ for any open $U \subset X$ is a quasi-isomorphism. This induces a quasiisomorphism of complexes of sheaves $\underline{\mathbb{C}} \rightarrow \Omega_{X}^{\bullet}$ on $X$ between the constant sheaf $\underline{\mathbb{C}}$ and $\Omega_{X}^{\bullet}$.

Proposition 15. The following diagram commutes in $D^{b}\left(\mathrm{Sh}_{\mathbb{C}}[X]\right)$

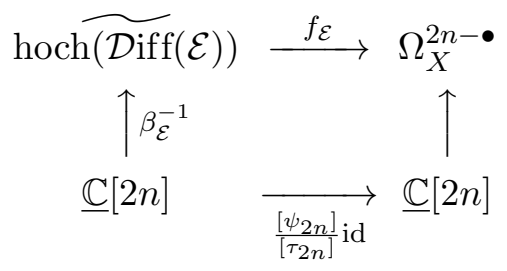

In particular, the map $f_{\mathcal{E}}$ in $D^{b}\left(\mathrm{Sh}_{\mathbb{C}}[X]\right)$ is independent of the choice of Fedosov connection on $\mathcal{B}_{\mathcal{E}}$.

Proof. As objects in $\mathrm{D}^{b}\left(\operatorname{Sh}_{\mathbb{C}}[X]\right)$, hoch $\left.\widetilde{(\mathcal{D i f f}}(\mathcal{E})\right)$ as well as $\Omega_{X}^{2 n-\bullet}$ are isomorphic to the shifted constant sheaf $\underline{\mathbb{C}}[2 n]$. Since $\mathbb{C}$ is an injective object in the category of sheaves of $\mathbb{C}$-vector spaces on $X$ (since it is flabby),

$$
\operatorname{Hom}_{\mathrm{D}^{b}\left(\mathrm{Sh}_{\mathbb{C}}[X]\right)}(\underline{\mathbb{C}}, \underline{\mathbb{C}}) \simeq \mathbb{C} .
$$


It follows that it is enough to verify this proposition for each open subset $U$ of $X$ with local holomorphic coordinates on which $\mathcal{E}$ is trivial. For such a $U$, the generalized trace map maps $c_{\mathcal{E}}(U)$ to a $2 n$-cycle mapping to the normalized Hochschild $2 n$-cycle

$$
r \sum_{\sigma \in S_{2 n}} \operatorname{sgn}(\sigma) \sigma\left(1, z_{1}, \frac{\partial}{\partial z_{1}}, \ldots, z_{n}, \frac{\partial}{\partial z_{n}}\right)
$$

It follows from Convention 1, Section 2.1 that $\beta_{\mathcal{E}}\left(c_{\mathcal{E}}(U)\right)=r$. Therefore, $\frac{1}{r} c_{\mathcal{E}}(U)$ is a $2 n$-cycle mapped to 1 by $\beta_{\mathcal{E}}$. It follows that $f_{\mathcal{E}}\left(\beta_{\mathcal{E}}^{-1}(1)\right)=\frac{1}{r} f_{\mathcal{E}}\left(c_{\mathcal{E}}(U)\right)=\frac{1}{r} \psi_{2 n}^{r}\left(c_{2 n}^{r}\right)$. The last equality is by Proposition 14. However $\psi_{2 n}^{r}\left(c_{2 n}^{r}\right)=\frac{\left[\psi_{2 n}^{r}\right]}{\left[\tau_{2 n}^{r}\right]} \tau_{2 n}^{r}\left(c_{2 n}^{r}\right)$. But $\frac{\left[\psi_{22}^{r}\right]}{\left[\tau_{2 n}^{r}\right]}=\frac{\left[\psi_{2 n}\right]}{\left[\tau_{2 n}\right]}$ by equation (1). Further, since $\tau_{2 n}\left(c_{2 n}\right)=1$ (see [11]), $\tau_{2 n}^{r}\left(c_{2 n}^{r}\right)=r$ by (1). Therefore, $\frac{1}{r} \psi_{2 n}^{r}\left(c_{2 n}^{r}\right)=\frac{\left[\psi_{2 n}\right]}{\left[\tau_{2 n}\right]}$. This proves the desired proposition.

Since $f_{\mathcal{E}, \psi_{2 n}}$ is a map of complexes of sheaves, the map

$$
\Gamma_{c}(X, \operatorname{hoch} \widetilde{(\mathcal{D i f f}}(\mathcal{E})) \rightarrow \mathbb{C}, \quad \alpha \mapsto \int_{X} f_{\mathcal{E}, \psi_{2 n}}(\alpha)
$$

descends to a (nonzero) linear functional on $\mathrm{H}_{0}\left(\Gamma_{c}(X, \operatorname{hoch} \widetilde{(\mathcal{D i f f}}(\mathcal{E}))\right)$. We shall denote this linear functional by $\int_{X} f_{\mathcal{E}, \psi_{2 n}}$.

3.4. Proof of Theorem 1. Proposition 15 implies that $\left[f_{\mathcal{E}, \psi_{2 n}}(\alpha)\right]=\frac{\left[\psi_{2 n}\right]}{\left[\tau_{2 n}\right]}[\alpha]$ in $\mathrm{H}_{c}^{2 n}(X, \mathbb{C})$ for any 0 -cycle $\alpha$ in $\Gamma_{c}(X, \operatorname{hoch(\mathcal {Diff}}(\mathcal{E}))$. Theorem 1 follows immediately from this observation and Theorem 4.

3.5. Remarks. $f_{\mathcal{E}, \psi_{2 n}}$ induces a map of complexes

$$
\mathrm{C} \bullet\left(\operatorname{Diff}^{\bullet}(\mathcal{E})\right) \rightarrow \Omega^{2 n-\bullet}(X) .
$$

This can equivalently be viewed as a family $\Theta_{i} \in \Omega^{i}\left(\mathrm{C}^{2 n-i}(\operatorname{Diff} \bullet(\mathcal{E}))\right)$ of cochain valued forms satisfying the differential equations

$$
d_{\mathrm{DR}} \Theta_{i}= \pm \delta \Theta_{i+1},
$$

where $\delta$ is the differential on the Hochschild cochain complex $\mathrm{C}^{2 n-i}\left(\operatorname{Diff}^{\bullet}(\mathcal{E})\right)$. In particular, $\Theta_{2 n}$ is a $2 n$-form with values in $\mathrm{C}^{0}\left(\operatorname{Diff}^{\bullet}(\mathcal{E})\right)$. When $X$ is compact, $\int_{X} \Theta_{2 n}$ is precisely the Hochschild 0-cocycle (11). This viewpoint seeing (11) as coming from "integrating $\psi_{2 n}^{r}$ over $X$ " is taken by [20].

More generally, there is a modified cyclic chain complex $\operatorname{Cycl(\mathcal {Diff}}(\mathcal{E}))$ related closely to hoch $\widetilde{\mathcal{D i f f}}(\mathcal{E}))$ (see Section 2.3). The construction of this section can be repeated for a continuous $G$-basic cyclic $2 n+2 p$-cocycle $\nu_{2 n+2 p}$ (see $[17,22]$ ). One obtains a map of complexes

$$
\left.\Gamma_{c}(X, \operatorname{Cycl} \widetilde{\operatorname{Diff}}(\mathcal{E}))\right) \rightarrow \Omega_{c}^{2 n+2 p-\bullet}(X)
$$

as a result. When $X$ is compact, and $k \geq p$, the above map on the $2 k$-th homology yields a map

$$
\mathrm{H}^{2 n-2 k}(X, \mathbb{C}) \oplus \mathrm{H}^{2 n-2 k+2}(X, \mathbb{C}) \oplus \cdots \oplus \mathrm{H}^{2 n}(X, \mathbb{C}) \rightarrow \mathrm{H}^{2 n+2 p-2 k}(X, \mathbb{C})
$$

It would be interesting to understand this map further. 


\section{Integrating the lifting formula $\Psi_{2 n+1}$}

We begin by outlining the construction of $\Psi_{2 n+1}$ in Section 4.1. Section 4.2 is devoted to proving Theorem 3.

Notation. For any algebra $A, \mathrm{M}_{r}(A)$ will denote the algebra of $r \times r$ matrices with entries in $A . \mathfrak{g l}_{\infty}^{\mathrm{fin}}(A)$ will denote the Lie algebra of finite matrices with entries in $A . \mathrm{M}_{\infty}(A)$ will denote the algebra of infinite matrices $M$ with entries in $A$ such that the $(i, j)$-th entry of $M$ vanishes whenever $|i-j|>C(M)$ where $C(M)$ is a constant (depending on $M$ ).

\subsection{About the lifting formula $\Psi_{2 n+1}$.}

4.1.1. The only explicit fact about the Lifting formula $\Psi_{2 n+1}$ that we shall use in this paper is its form. Let $\Psi \operatorname{Diff}_{n}$ denote the algebra $\mathbb{C}\left[\left[y_{1}, \ldots, y_{n}\right]\right]\left[y_{1}^{-1}, \ldots, y_{n}^{-1}\right]\left[\left[\partial_{1}^{-1}, \ldots, \partial_{n}^{-1}\right]\right]\left[\partial_{1}, \ldots, \partial_{n}\right]$ $\left(\partial_{i}:=\frac{\partial}{\partial y_{i}}\right)$ of formal pseudodifferential operators. The coefficient at $y_{1}^{-1} \cdots y_{n}^{-1} \partial_{1}^{-1} \cdots \partial_{n}^{-1}$ defines a linear functional on $\Psi \operatorname{Diff}_{n}$. This functional vanishes on commutators of elements of $\Psi$ Diff $_{n}$. We therefore call it a trace and denote it by $\operatorname{Tr}_{\Psi \operatorname{Diff}}: \Psi \operatorname{Diff}_{n} \rightarrow \mathbb{C}$ (see [1]). Recall that the "usual" matrix trace $\operatorname{Tr}_{\mathfrak{g l}}$ fin yields a linear map from $\mathfrak{g l}_{\infty}^{\mathrm{fin}}(A)$ to $A$ for any algebra $A$. It can then be verified that $\operatorname{Tr}_{\Psi \operatorname{Diff}_{n}} \circ \operatorname{Tr}_{\mathfrak{g l}_{\infty}^{\mathrm{fin}}}$ yields a trace on the algebra $\mathfrak{g l}_{\infty}^{\mathrm{fin}}\left(\Psi \operatorname{Diff} f_{n}\right)$.

For any $\alpha \in A$, let $\alpha \otimes 1_{\infty}$ denote the diagonal matrix $\alpha \in \mathrm{M}_{\infty}(A)$. Let $D_{i}, 1 \leq i \leq 2 n$ be the derivations on $\mathfrak{g l}_{\infty}^{\mathrm{fin}}\left(\Psi \operatorname{Diff} f_{n}\right)$ given by $D_{i}=\operatorname{ad}\left(\ln \left(x_{i}\right) \otimes 1_{\infty}\right)$ for $1 \leq i \leq n$ and $D_{i}=$ $\operatorname{ad}\left(\ln \left(\partial_{i-n}\right) \otimes 1_{\infty}\right)$ for $n+1 \leq i \leq 2 n$. We recall from [21] that $\left[D_{i}, D_{j}\right]=\operatorname{ad}\left(Q_{i j} \otimes 1_{\infty}\right)$ for some elements $Q_{i j}$ of $\Psi \operatorname{Diff}_{n}$.

Consider the set $\mathcal{S}$ of all markings of the interval [1,2n-1] such that

(i) Only finitely many integral points are marked.

(ii) The distance between any two distinct marked points is at least 2 .

Note that the "empty" marking where no point is marked is also an element of $\mathcal{S}$.

Let $t \in S$ be a marking of $[1,2 n-1]$ marking the integers $i_{1}, \ldots, i_{k}$. Define

$$
\mathcal{O}(t)\left(A_{1}, \ldots, A_{2 n+1}\right)=\operatorname{Alt}_{A, D}\left(\operatorname{Tr}_{\Psi \operatorname{Diff}} \circ \operatorname{Tr}_{\mathfrak{g l}_{\infty}^{\text {fin }}}\left(P_{1, t} \circ \cdots \circ P_{2 n+1, t}\right)\right),
$$

where

if $j$ is marked, $P_{j, t}=A_{j} Q_{j, j+1}$ and $P_{j+1, t}=A_{j+1}$,

$P_{j, t}=D_{j}\left(A_{j}\right)$ if $j$ and $j-1$ are not marked and $j \neq 2 n+1$,

$P_{2 n+1, t}=A_{2 n+1}$.

Note that 0 and $2 n$ should be thought of as unmarked by default in the above formula. If $t$ is the "empty" marking,

$$
\mathcal{O}(t)\left(A_{1}, \ldots, A_{2 n+1}\right)=\operatorname{Alt}_{A, D}\left(\operatorname{Tr}_{\Psi \operatorname{Diff}_{n}} \circ \operatorname{Tr}_{\mathfrak{g r}_{\infty}^{\mathrm{fin}}}\left(D_{1}\left(A_{1}\right) \cdots D_{2 n}\left(A_{2 n}\right) A_{2 n+1}\right)\right) .
$$

Theorem 6 ([21]). The linear functional

$$
\left(A_{1}, \ldots, A_{2 n+1}\right) \mapsto \sum_{t \in \mathcal{S}} \mathcal{O}(t)\left(A_{1}, \ldots, A_{2 n+1}\right)
$$

is a $2 n+1$-cocycle in $\mathrm{C}_{\text {Lie }}^{2 n+1}\left(\mathfrak{g l}_{\infty}^{\text {fin }}\left(\Psi \operatorname{Diff}_{n}\right) ; \mathbb{C}\right)$.

The natural inclusion of algebras $\operatorname{Diff}_{n} \subset \Psi \operatorname{Diff}_{n}$ extends to an inclusion $\mathfrak{g l}_{\infty}^{\text {fin }}\left(\operatorname{Diff}_{n}\right) \subset$ $\mathfrak{g l}_{\infty}^{\mathrm{fin}}\left(\Psi \operatorname{Diff}_{n}\right)$. The formula from Theorem 6 therefore, gives us the cocycle

$$
\Psi_{2 n+1} \in \mathrm{C}_{\text {Lie }}^{2 n+1}\left(\mathfrak{g l}_{\infty}^{\mathrm{fin}}\left(\operatorname{Diff}_{n}\right), \mathbb{C}\right) .
$$

$\Psi_{2 n+1}$ is referred to as a lifting formula. 
4.1.2. Note that for any algebra $A$, we have isomorphisms

$$
\mathrm{M}_{m}(A) \otimes \mathrm{M}_{r}(\mathbb{C}) \simeq \mathrm{M}_{r m}(A)
$$

of algebras. Taking the direct limit of these isomorphisms, we obtain a map of algebras (and therefore, Lie algebras)

$$
i_{m}: \quad \mathfrak{g l}_{\infty}^{\mathrm{fin}}\left(\mathrm{M}_{m}(A)\right) \simeq \mathrm{M}_{m}(A) \otimes \mathfrak{g l}_{\infty}^{\mathrm{fin}}(\mathbb{C}) \rightarrow \mathfrak{g l}_{\infty}^{\mathrm{fin}}(A)
$$

It follows that $\Psi_{2 n+1}$ yields a cocycle in $\mathrm{C}_{\text {lie }}^{2 n+1}\left(\mathfrak{g l}_{\infty}^{\mathrm{fin}}\left(\mathrm{M}_{m}\left(\operatorname{Diff}_{n}\right)\right), \mathbb{C}\right)$ as well. We denote this cocycle by $\Psi_{2 n+1}^{m}$.

For $M \in \mathrm{M}_{m}\left(\operatorname{Diff}_{n}\right), M \otimes$ id shall refer to the element $i_{m}(M \otimes \mathrm{id})$ of $\mathrm{M}_{\infty}\left(\operatorname{Diff}_{n}\right)$. For any $p>m$, let $\iota_{m, p}: \mathrm{M}_{m}\left(\right.$ Diff $\left._{n}\right) \rightarrow \mathrm{M}_{p}\left(\right.$ Diff $\left._{n}\right)$ denote the natural embedding obtained by "padding with zeros".

Proposition 16. If $A_{1}, \ldots, A_{2 n}, M \in \mathrm{M}_{m}\left(\operatorname{Diff}_{n}\right)$ and $B_{1}, \ldots, B_{2 n} \in \mathrm{M}_{p}\left(\operatorname{Diff}_{n}\right)$ then

$$
\begin{gathered}
\Psi_{2 n+1}^{m+p}\left(\left(A_{1} \oplus B_{1}\right) \otimes \mathrm{id}, \ldots,\left(A_{2 n} \oplus B_{2 n}\right) \otimes \mathrm{id}, \iota_{m, m+p}(M) \otimes E_{1,1}\right) \\
=\Psi_{2 n+1}^{m}\left(A_{1} \otimes \mathrm{id}, \ldots, A_{2 n} \otimes \mathrm{id}, M \otimes \mathrm{E}_{1,1}\right) .
\end{gathered}
$$

Proof. It is enough to show that for any $t \in \mathcal{S}$,

$$
\begin{aligned}
& \mathcal{O}(t)\left(\left(A_{1} \oplus B_{1}\right) \otimes \mathrm{id}, \ldots,\left(A_{2 n} \oplus B_{2 n}\right) \otimes \mathrm{id}, \iota_{m, m+p}(M) \otimes \mathrm{E}_{1,1}\right) \\
& \quad=\mathcal{O}(t)\left(A_{1} \otimes \mathrm{id}, \ldots, A_{2 n} \otimes \mathrm{id}, M \otimes \mathrm{E}_{1,1}\right) .
\end{aligned}
$$

The summands on the left hand side of (12) are of the form $\operatorname{Tr}_{\Psi \operatorname{Diff}} \circ \operatorname{Tr}_{\mathfrak{g l}_{\infty}^{\text {fin }}}\left(X_{1} \circ \cdots \circ X_{r}\right)$ where $X_{i}=\left(A_{j} \oplus B_{j}\right) \otimes$ id or $X_{i}=\iota_{m, m+p}(M) \otimes \mathrm{E}_{1,1}$ or $X_{i}=\lambda \otimes$ id for some element $\lambda$ of $\Psi$ Diff ${ }_{n}$. It is easy to see that this summand does not change if each $X_{i}$ of the form $X_{i}=\left(A_{j} \oplus B_{j}\right) \otimes$ id is replaced by $A_{j} \otimes \mathrm{id}$. Doing this however transforms the sum on the left hand side to that on the right hand side.

\subsection{Constructing Shoikhet's holomorphic noncommutative residue in general.}

4.2.1. Let $\mathfrak{g}$ be a DG-Lie algebra. Then, $\left\{C_{p, q}:=\left(\wedge^{p} \mathfrak{g}\right)^{-q} \mid p \geq 1\right\}$ becomes a double complex whose horizontal differential is the Chevalley-Eilenberg differential and whose vertical differential is that induced by the differential intrinsic to $\mathfrak{g}$. We denote the complex $\operatorname{Tot}^{\oplus}\left(C_{\bullet, \bullet}\right)$ by $C_{\bullet}^{\text {lie }}(\mathfrak{g})$. Similarly we denote $\operatorname{Tot}^{\Pi}\left(C_{\bullet}, \bullet\right)$ by $C_{\bullet}^{\Pi, l i e}(\mathfrak{g})$. There is a natural map of complexes from $C_{\bullet}^{\text {lie }}(\mathfrak{g})$ to $C_{\bullet}^{\Pi, l i e}(\mathfrak{g})$. Let $\omega \in \mathfrak{g}^{1}$ be a Maurer-Cartan element. Let $\mathfrak{g}_{\omega}$ denote the twisted Lie algebra whose underlying differential is $d+[\omega,-]$.

Proposition 17. There is a natural map of complexes

$$
\begin{aligned}
C_{\bullet}^{\Pi, \text { lie }}\left(\mathfrak{g}_{\omega}\right) & \rightarrow C_{\bullet}^{\Pi, \text { lie }}(\mathfrak{g}), \\
\left(g_{0}, \ldots, g_{k}\right) & \mapsto \sum_{j \geq 0} \frac{1}{j !}\left(g_{0}, \ldots, g_{k}, \omega, \omega, \ldots, \omega\right) \quad\left(j \quad \omega^{\prime} s\right) .
\end{aligned}
$$

Proof. Since this proposition is completely analogous to Proposition 2.4 of [10], we shall only sketch the proof. Denote the differential of $\mathfrak{g}$ by $d$. Let $d_{\mathrm{CE}}$ denote the Chevalley-Eilenberg differential. Let $(\omega)^{j}:=\omega \wedge \cdots \wedge \omega j$ times.

Step 1. One first notes that

$$
d_{\mathrm{CE}}(\omega)^{j}=\frac{j(j-1)}{2}[\omega, \omega] \wedge(\omega)^{j-2}=-j(j-1) d \omega \wedge(\omega)^{j-2}=-j d\left(\omega^{j-1}\right) .
$$


The middle equality above is because $\omega$ is a Maurer-Cartan element. It follows that if $\phi_{j}:=$ $\frac{1}{j !}(\omega)^{j}$, then

$$
d_{\mathrm{CE}} \phi_{j}=-d \phi_{j-1} .
$$

Step 2. Let $g_{0}, \ldots, g_{k}$ be homogenous elements of $\mathfrak{g}$. Let $d_{i}$ denote the degree of $g_{i}$. Let $\mathcal{G}:=g_{0} \wedge \cdots \wedge g_{k}$. One then verifies (by a direct calculation) that

$$
d_{\mathrm{CE}}\left(\mathcal{G} \wedge \phi_{j}\right)=\left(d_{\mathrm{CE}} \mathcal{G}\right) \wedge \phi_{j}+(-1)^{k+1} \mathcal{G} \wedge d_{\mathrm{CE}} \phi_{j}+(-1)^{d_{0}+\cdots+d_{k}+k+1}(\operatorname{ad}(\omega) \mathcal{G}) \wedge \phi_{k-1} .
$$

The desired proposition follows from equations (13) and (14) after inserting the relevant definitions and summing over $k$.

Notation. In some situations in this section, we find it better to specify the differential of a DG-Lie algebra: if $d$ is the differential on a DG-Lie algebra $\mathfrak{g}$ we often denote $C_{\bullet}(\mathfrak{g})$ and $\mathrm{C}_{\bullet}^{\Pi, l i e}(\mathfrak{g})$ by $\mathrm{C}_{\bullet}^{\text {lie }}(\mathfrak{g}, d)$ and $\mathrm{C}_{\bullet}^{\Pi, \text { lie }}(\mathfrak{g}, d)$ respectively.

4.2.2. Let $D$ be the Fedosov differential on $\Omega^{\bullet}\left(\mathcal{B}_{\mathcal{E}}\right)$ chosen as in Section 3.1.4. This choice ensures that there is a morphism $\mathcal{D} \operatorname{iff}^{\bullet}(\mathcal{E}) \rightarrow \Omega^{\bullet}\left(\mathcal{B}_{\mathcal{E}}\right)$ of sheaves of DG-algebras on $X$. Let $U$ be an open subset of $X$ on which $J_{1} \mathcal{E}$ is trivial. Recall that any trivialization of $J_{1} \mathcal{E}$ on an open subset $U$ of $X$ induces an term by term isomorphism of sheaves DG-algebras $\left(\Omega^{\bullet}\left(\mathcal{B}_{\mathcal{E}}\right)_{U}, D\right) \rightarrow$ $\left(\Omega_{U}^{\bullet}\left(\mathrm{M}_{r}\left(\operatorname{Diff}_{n}\right)\right), d+[\omega,-]\right)$. Also, $\omega \otimes 1_{N}$ is a Maurer-Cartan element in $\mathfrak{g l}_{\infty}^{\mathrm{fin}}\left(\Omega^{\bullet}\left(U, \mathrm{M}_{r}\left(\operatorname{Diff}_{n}\right)\right)\right)$ for any sufficiently large natural number $N$. Here $1_{N}$ denotes the $N \times N$ identity matrix "padded with 0 's" on the right and bottom to obtain an element of $\mathfrak{g l}_{\infty}^{\mathrm{ffin}}(\mathbb{C})$. We will continue to denote this element by $\omega$ for notational brevity.

One therefore has the following composite map of complexes

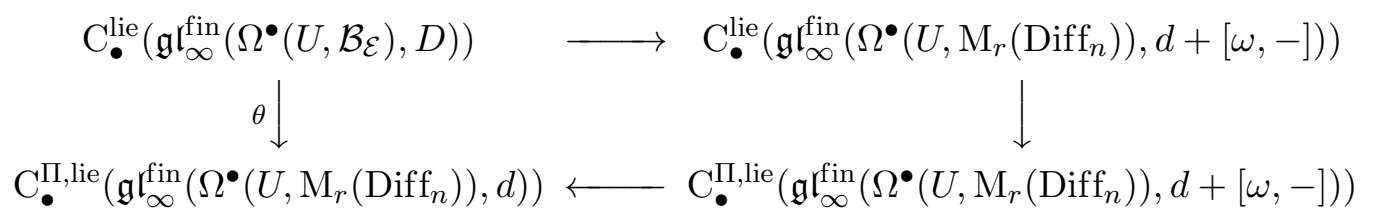

The horizontal arrow on top is from the isomorphism of $\left(\Omega^{\bullet}\left(U, \mathcal{B}_{\mathcal{E}}\right), D\right)$ with $\left(\Omega^{\bullet}\left(U, \mathrm{M}_{r}\left(\operatorname{Diff}_{n}\right)\right), d+\right.$ $[\omega,-])$ induced by $\varphi$. The vertical arrow on the right is the natural map mentioned in Section 4.2.1. The horizontal arrow on the bottom is from Proposition 17. Let $\Xi_{2 n+1}$ be any continuous $2 n+1$ cocycle in $\mathrm{C}_{\text {lie }}^{2 n+1}\left(\mathfrak{g f f}_{\infty}^{\mathrm{ffin}}\left(\mathrm{M}_{r}\left(\operatorname{Diff}_{n}\right)\right), \mathbb{C}\right)$. As in Proposition 11, Section 3.2.3, evaluation at $\Xi_{2 n+1}$ yields a map of complexes from $\mathrm{C}_{\bullet}^{\Pi, l i e}\left(\mathfrak{g}_{\infty}^{\mathrm{ffin}}\left(\Omega^{\bullet}\left(U, \mathrm{M}_{r}\left(\operatorname{Diff}_{n}\right)\right), d\right)\right)[1]$ to $\Omega^{2 n-\bullet}(U)$ (with the differential on $\Omega^{2 n-p}$ being $(-1)^{p} d_{\mathrm{DR}}$ ). Composing this map with $\theta$, and applying the involution multiplying a $p$-form by $(-1)^{\left\lfloor\frac{p}{2}\right\rfloor}$, we obtain a map of complexes

$$
\left(\varphi, \Xi_{2 n+1}\right)_{*}: \quad \mathrm{C}_{\bullet}^{\text {lie }}\left(\mathfrak{g l}_{\infty}^{\mathrm{fin}}\left(\Omega^{\bullet}\left(U, \mathcal{B}_{\mathcal{E}}\right), D\right)\right)[1] \rightarrow \Omega^{2 n-\bullet}(U) .
$$

Explicitly, if $\mu \in \mathrm{C}_{p}^{\text {lie }}\left(\mathfrak{g l f}_{\infty}^{\mathrm{ffin}}\left(\Omega^{\bullet}\left(U, \mathcal{B}_{\mathcal{E}}\right), D\right)\right)$,

$$
\left(\varphi, \Xi_{2 n+1}\right)_{*}(\mu)=(-1)^{\left\lfloor\frac{2 n-p}{2}\right\rfloor} \sum_{k} \frac{1}{k !} \Xi_{2 n+1}\left(\hat{\mu} \wedge \omega^{k}\right),
$$

where $\hat{\mu}$ is the image of $\mu$ in $\mathrm{C}_{p}^{\text {lie }}\left(\mathfrak{g l}_{\infty}^{\mathrm{fin}}\left(\Omega^{\bullet}\left(U, \mathrm{M}_{r}\left(\operatorname{Diff}_{n}\right)\right), d+[\omega,-]\right)\right)$. In particular, we obtain a map of complexes

$$
\mathrm{C}_{\bullet}^{\mathrm{lie}}\left(\Gamma\left(U, \mathfrak{g l}_{\infty}^{\mathrm{fin}}(\mathcal{D i f f} \bullet(\mathcal{E}))\right)\right)[1] \rightarrow \Omega^{2 n-\bullet}(U) .
$$

Proposition 18. The above map extends to a map

$$
\lambda\left(\Xi_{2 n+1}\right): \widetilde{\operatorname{Lie}}(\mathcal{D} \operatorname{iff}(\mathcal{E}))[1] \rightarrow \Omega_{U}^{2 n-\bullet}
$$

of complexes of sheaves of $\mathbb{C}$-vector spaces on $U$. 
Proof. This follows from the continuity of $\Xi_{2 n+1}$. The argument proving this is completely analogous to the proof of Proposition 13. However, since $\Xi_{2 n+1}$ may not be $G:=G L(n, \mathbb{C}) \times$ $G L(r, \mathbb{C})$-basic, we can only guarantee the existence of a map of complexes of sheaves over $U$.

Corollary 2. If $X$ is complex parallelizable and $\mathcal{E}$ is trivial, $\lambda\left(\Xi_{2 n+1}\right)$ yields a map of complexes of sheaves $\widetilde{\operatorname{Lie}}(\mathcal{D} \operatorname{iff}(\mathcal{E}))[1] \rightarrow \Omega_{X}^{2 n-\bullet}$ on $X$.

Proof. The obstruction to globalizing $\lambda\left(\Xi_{2 n+1}\right)$ comes from the fact that there is no consistent way of choosing a section of $J_{1} \mathcal{E}$ over $X$ in general.

Remark 2. If $\Xi_{2 n+1}$ is $G L(n) \times G L(r)$-basic, $\lambda\left(\Xi_{2 n+1}\right)$ yields a map of complexes of sheaves $\widetilde{\operatorname{Lie}}(\mathcal{D} \operatorname{iff}(\mathcal{E}))[1] \rightarrow \Omega_{X}^{2 n-\bullet}$ for arbitrary (not necessarily complex parallelizable) $X$ and arbitrary (not necessarily trivial) $\mathcal{E}$. The proof of this assertion is completely analogous to that of Proposition 12 .

\subsubsection{Properties of $\lambda\left(\Xi_{2 n+1}\right)$.}

Proposition 19. For a fixed trivialization of $J_{1} \mathcal{E}$, the map induced by $\lambda\left(\Xi_{2 n+1}\right)$ in $D^{b}\left(\mathrm{Sh}_{\mathbb{C}}[U]\right)$ depends only on the class of $\Xi_{2 n+1}$ in $\mathrm{H}_{\text {lie }}^{2 n+1}\left(\mathrm{M}_{r}\left(\operatorname{Diff}_{n}\right)\right)$.

Proof. We will show that if $\Xi_{2 n+1}$ is a coboundary, then $\left(\varphi, \Xi_{2 n+1}\right)_{*}$ is null-homotopic. The homotopy we shall provide will automatically yield a homotopy between $\lambda\left(\Xi_{2 n+1}\right)$ and 0 . Recall that the map $\left(\varphi, \Xi_{2 n+1}\right)_{*}$ was obtained by composing the map $\theta$ from the commutative diagram (15) with evaluation at $\Xi_{2 n+1}$ followed by multiplying $p$-forms by $(-1)^{\left\lfloor\frac{p}{2}\right\rfloor}$. If $\Xi_{2 n+1}=d \beta$, evaluation at $\beta$ gives a homotopy between evaluation at $\Xi_{2 n+1}$ and 0 . This proves that $\left(\varphi, \Xi_{2 n+1}\right)_{*}$ is null-homotopic when $\Xi_{2 n+1}=d \beta$. Explicitly, if $\mu \in$

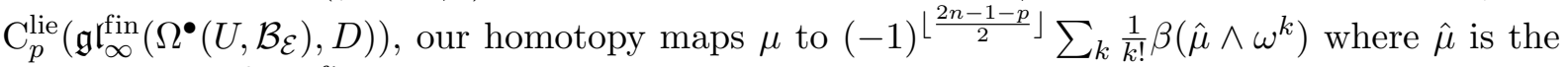
image of $\mu$ in $\mathrm{C}_{p}^{\text {lie }}\left(\mathfrak{g l}_{\infty}^{\mathrm{fin}}\left(\Omega^{\bullet}\left(U, \mathrm{M}_{r}\left(\operatorname{Diff}_{n}\right)\right), d+[\omega,-]\right)\right)$. It is easy to see that since $\beta$ is a continuous cochain, our homotopy yields a homotopy between $\lambda(d \beta)$ and the 0 map.

Proposition 20. The map induced by $\lambda\left(\Xi_{2 n+1}\right)$ in $D^{b}\left(\mathrm{Sh}_{\mathbb{C}}[U]\right)$ is independent of the trivialization of $J_{1} \mathcal{E}$ chosen.

Proof. Let $\mathfrak{h}$ be a Lie subalgebra of $\mathfrak{g}$. Let $\mathrm{H}_{\text {lie }}^{\bullet}(\mathfrak{g}, \mathfrak{h})$ denote the Lie algebra cohomology of $\mathfrak{g}$ relative to $\mathfrak{h}$ (with coefficients in the trivial module). The proof of Lemma 5.2 of [11] goes through with minor modifications to show that

$$
\mathrm{H}_{\text {lie }}^{2 n+1}\left(\mathfrak{g l}_{\infty}^{\mathrm{fin}}\left(\mathrm{M}_{r}\left(\operatorname{Diff}_{n}\right)\right), \mathfrak{g l}_{\infty}^{\mathrm{fin}} \oplus \mathfrak{g l}_{n} \oplus \mathfrak{g l}_{r}\right) \simeq \mathrm{H}_{\text {lie }}^{2 n+1}\left(\mathfrak{g l}_{\infty}^{\mathrm{fin}}\left(\mathrm{M}_{r}\left(\operatorname{Diff}_{n}\right)\right)\right) .
$$

It follows that there exists a $G$-basic cocycle $\Theta_{2 n+1}$ such that $\left[\Theta_{2 n+1}\right]=\left[\Xi_{2 n+1}\right]$ in $\mathrm{H}_{\text {lie }}^{2 n+1}\left(\mathfrak{g l}_{\infty}^{\mathrm{fin}}\left(\mathrm{M}_{r}\left(\operatorname{Diff}_{n}\right)\right)\right)$. By Proposition $19, \lambda\left(\Xi_{2 n+1}\right)=\lambda\left(\Theta_{2 n+1}\right)$ as a morphism in $\mathrm{D}^{b}\left(\mathrm{Sh}_{\mathbb{C}}[U]\right)$. The proof that $\lambda\left(\Theta_{2 n+1}\right)$ is independent of the chosen trivialization of $J_{1} \mathcal{E}$ (as a morphism in $\left.\mathrm{D}^{b}\left(\mathrm{Sh}_{\mathbb{C}}[U]\right)\right)$ is identical that of Proposition 12 (with the obvious modifications).

Proposition 21. The map induced by $\lambda\left(\Xi_{2 n+1}\right)$ in $D^{b}\left(\mathrm{Sh}_{\mathbb{C}}[U]\right)$ is independent of the Fedosov differential $D$ on $\Omega^{\bullet}\left(\mathcal{B}_{\mathcal{E}}\right)$ (for a fixed trivialization of $J_{1} \mathcal{E}$ over $U$ ).

Proof. From the discussion in Section 2.4.1, $\widetilde{\operatorname{Lie}}(\operatorname{Diff}(\mathcal{E}))[1]$ is isomorphic to $\mathbb{C}[2 n] \oplus V$ in $\mathrm{D}^{b}\left(\mathrm{Sh}_{\mathbb{C}}[U]\right)$ where $V$ is a direct sum of shifted constant sheaves concentrated in homological degree $\geq 2 n+2$. Also, $\Omega^{2 n-\bullet}$ is isomorphic to $\underline{\mathbb{C}}[2 n]$ in $\mathrm{D}^{b}\left(\mathrm{Sh}_{\mathbb{C}}[U]\right)$. Since $\underline{\mathbb{C}}$ is an injective object in the category of sheaves of $\mathbb{C}$-vector spaces, $\operatorname{Hom}_{\mathrm{D}^{b}\left(\mathrm{Sh}_{\mathbb{C}}[U]\right)}(V, \mathbb{C}[2 n])=0$. It therefore, suffices to show that $\lambda\left(\Xi_{2 n+1}\right)$ applied to a fixed $2 n$ cycle generating the $2 n$-st homology of $\Gamma(U, \widetilde{\operatorname{Lie}}(\mathcal{D} \operatorname{iff}(\mathcal{E}))[1])$ is independent of the choice of Fedosov differential. 
In fact, since $J_{1} \mathcal{E}$ is trivial over $U$, the $2 n$-th homology of $\Gamma(U, \widetilde{\operatorname{Lie}}(\mathcal{D} \operatorname{iff}(\mathcal{E}))[1])$ is in fact generated by a $2 n$-cycle $c_{\mathcal{E}}^{\text {lie }}(U)$ in $\widehat{\mathrm{C}}_{\bullet}^{\text {lie }}\left(\Gamma\left(U, \mathfrak{g r}_{\infty}^{\mathrm{fin}}(\mathcal{D} \operatorname{iff}(\mathcal{E}))\right)\right)[1]$ (see Section 2.4.1).

As in the proof of Proposition 14, given any ( $G$-equivariant) section $\varphi: J_{1} \mathcal{E} \rightarrow J_{\infty} \mathcal{E}$, one has a composite map of complexes

$$
\widehat{\mathrm{C}}_{\bullet}^{\mathrm{lie}}\left(\Gamma\left(U, \mathfrak{g l}_{\infty}^{\mathrm{fin}}(\mathcal{D i f f}(\mathcal{E}))\right)\right)[1] \rightarrow \Omega^{2 n-\bullet, 0}\left(\left.J_{\infty} \mathcal{E}\right|_{U}\right) \rightarrow \Omega^{2 n-\bullet}\left(J_{1} \mathcal{E}\right) \rightarrow \Omega^{2 n-\bullet}(U) .
$$

The "restriction" of the first map above to $C_{\bullet}^{\text {lie }}\left(\Gamma\left(U, \mathfrak{g l}_{\infty}^{\text {fin }}(\mathcal{D} \operatorname{iff}(\mathcal{E}))\right)\right)[1]$ is constructed just like the analogous map in the proof of Proposition 14. The middle arrow is $\varphi^{*}$ where $\varphi: J_{1} \mathcal{E} \rightarrow J_{\infty} \mathcal{E}$. The third is the pullback by the chosen section of $J_{1} \mathcal{E}$. Since $\varphi$ is unique upto homotopy, the image of $c_{\mathcal{E}}^{\text {lie }}(U)$ under the composite map (17) is independent of $\varphi$. Finally, Proposition 8 tells us that if $\varphi$ is the section associated with the Fedosov connection $D$ on $\mathcal{B}_{\mathcal{E}}$ (as in Section 3.1.5), the image of $c_{\mathcal{E}}^{\text {lie }}(U)$ under the composite map (17) coincides with the image of $c_{\mathcal{E}}^{\text {lie }}(U)$ under the "restriction" of $\lambda\left(\Xi_{2 n+1}\right)$ to $\widehat{\mathrm{C}}_{\bullet}^{\text {lie }}\left(\Gamma\left(U, \mathfrak{g l}_{\infty}^{\mathrm{fin}}(\mathcal{D} \operatorname{iff}(\mathcal{E}))\right)\right)[1]$. This proves the desired proposition.

Propositions 20 and 21 show that the map induced by $\lambda\left(\Xi_{2 n+1}\right)$ in $\mathrm{D}^{b}\left(\mathrm{Sh}_{\mathbb{C}}[U]\right)$ is independent of the choices made to define it. Let $\mathcal{F}$ be another holomorphic vector bundle on $X$. Let $\iota$ denote the natural map of sheaves of DG-algebras between $\mathcal{D} \operatorname{iff} \bullet(\mathcal{E})$ and $\operatorname{Diff}^{\bullet}(\mathcal{E} \oplus \mathcal{F})$. Note that $\iota$ induces a map of sheaves $\bar{\iota}: \widetilde{\operatorname{Lie}}(\mathcal{D} \operatorname{iff}(\mathcal{E}))[1] \rightarrow \widetilde{\operatorname{Lie}}(\mathcal{D} \operatorname{iff}(\mathcal{E} \oplus \mathcal{F}))[1]$ on $X$. Let $r$ and $s$ be the ranks of $\mathcal{E}$ and $\mathcal{F}$ respectively. Recall that for every positive integer $m$ we have the lifting cocycle $\Psi_{2 n+1}^{m}$ described in Section 4.1.2 $\left(\Psi_{2 n+1}^{1}=\Psi_{2 n+1}\right)$.

Proposition 22. The following diagram commutes in $D^{b}\left(\mathrm{Sh}_{\mathbb{C}}[U]\right)$

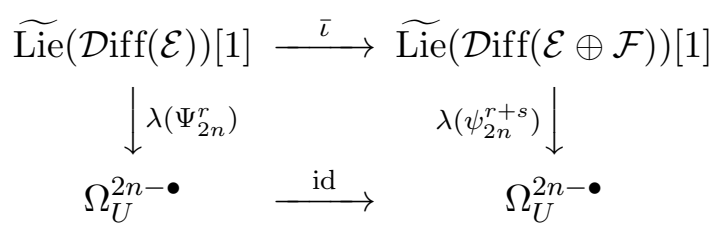

Proof. Recall that there is a natural map of complexes from $\widehat{\mathrm{C}}_{\bullet}^{\text {lie }}\left(\Gamma\left(U, \mathfrak{g l}_{\infty}^{\mathrm{fin}}(\mathcal{D} \operatorname{iff}(\mathcal{E}))\right)\right)[1]$ to $\Gamma(U, \widetilde{\operatorname{Lie}}(\mathcal{D} \operatorname{iff}(\mathcal{E}))[1])$ (and similarly for $\mathcal{E} \oplus \mathcal{F})$. As observed while proving Proposition 5, this map is an isomorphism on homology, and the constant sheaf of $U$ corresponding to the homology of $\widehat{\mathrm{C}}_{\bullet}^{\text {lie }}(\Gamma(U, \mathcal{D} \operatorname{iff}(\mathcal{E})))[1]$ is isomorphic to $\widetilde{\operatorname{Lie}}(\mathcal{D} \operatorname{iff}(\mathcal{E}))[1]$ in $\mathrm{D}^{b}\left(\operatorname{Sh}_{\mathbb{C}}[U]\right)$. It therefore, suffices to show that the following diagram commutes upto homology

$$
\begin{aligned}
& \widehat{\mathrm{C}}_{\bullet}^{\text {lie }}\left(\Gamma\left(U, \mathfrak{g l}_{\infty}^{\mathrm{fin}}(\mathcal{D} \operatorname{iff}(\mathcal{E}))\right)\right)[1] \stackrel{\bar{\iota}}{\longrightarrow} \widehat{\mathrm{C}}_{\bullet}^{\mathrm{lie}}\left(\Gamma\left(U, \mathfrak{g l}_{\infty}^{\mathrm{fin}}(\mathcal{D} \operatorname{iff}(\mathcal{E} \oplus \mathcal{F}))\right)\right)[1] \\
& \downarrow \lambda\left(\Psi_{2 n}^{r}\right) \quad \lambda\left(\psi_{2 n}^{r+s}\right) \downarrow \\
& \Omega_{U}^{2 n-\bullet} \quad \stackrel{\text { id }}{\longrightarrow} \quad \Omega_{U}^{2 n-\bullet}
\end{aligned}
$$

By arguments paralleling the proofs of Propositions 20 and 21, we are free to choose the sections $\left.U \rightarrow J_{\infty} \mathcal{E}\right|_{U}$ and $\left.U \rightarrow J_{\infty}(\mathcal{E} \oplus \mathcal{F})\right|_{U}$ that we shall use. Fix holomorphic coordinates $z_{1}, \ldots, z_{n}$ on $U$. Fix a trivialization of $\mathcal{E}$ over $U$. Also fix a trivialization of $\mathcal{F}$ over $U$. If $\left\{e_{i}\right\}$ and $\left\{f_{j}\right\}$ are the ordered bases of $\Gamma(U, \mathcal{E})$ and $\Gamma(U, \mathcal{F})$ (over $\Gamma(U, \mathcal{O})$ ) we have chosen in our trivializations, we trivialize $\mathcal{E} \oplus \mathcal{F}$ by choosing the ordered basis $\left\{e_{1}, \ldots, e_{r}, f_{1}, \ldots, f_{s}\right\}$ of $\Gamma(U, \mathcal{E} \oplus \mathcal{F})$. With these choices, we obtain sections of $J_{1} \mathcal{E}$ and $J_{1}(\mathcal{E} \oplus \mathcal{F})$ over $U$. The chosen coordinate system and trivialization of $\mathcal{E}$ identify $\Gamma(U, \mathcal{D} \operatorname{iff}(\mathcal{E}))$ with $\mathrm{M}_{r}(\operatorname{Diff}(U))$. The (G-equivariant) section of $J_{\infty} \mathcal{E}$ we choose is the one that maps $\frac{\partial}{\partial z_{i}}$ to $\frac{\partial}{\partial y_{i}}$ and $f\left(z_{1}, \ldots, z_{n}\right)$ to

$$
\left.\left(a_{1}, \ldots, a_{n}\right) \mapsto \sum_{I} \frac{\partial f}{\partial z^{I}}\right|_{\left(a_{1}, \ldots, a_{n}\right)} y^{I} .
$$


Let $D \in \Gamma\left(U, \mathfrak{g l} \mathfrak{l}_{\infty}^{\mathrm{fin}}(\mathcal{D} \operatorname{iff}(\mathcal{E}))\right)$ be arbitrary. Let $\hat{D}$ denote the flat section of $\mathrm{C}^{\infty}\left(U, \mathfrak{g l}_{\infty}^{\mathrm{fin}}\left(\mathrm{M}_{r}\left(\operatorname{Diff}_{n}\right)\right)\right)$ corresponding to $D$. Note that by our choices of sections of $J_{\infty} \mathcal{E}$ and $J_{\infty}(\mathcal{E} \oplus \mathcal{F})$,

$$
\widehat{\iota(D)}=\iota_{r, r+s} \hat{D} .
$$

It follows from equation (16) and Proposition 16 that the following diagram commutes literally (not just upto homology)

$$
\begin{array}{ccc}
\mathrm{C}_{\bullet}^{\mathrm{lie}}\left(\Gamma\left(U, \mathfrak{g l}_{\infty}^{\mathrm{fin}}(\mathcal{D i f f}(\mathcal{E}))\right)\right)[1] & \stackrel{\bar{\iota}}{\longrightarrow} & \mathrm{Cl}_{\bullet}^{\mathrm{lie}}\left(\Gamma\left(U, \mathfrak{g l}_{\infty}^{\mathrm{fin}}(\mathcal{D i f f}(\mathcal{E} \oplus \mathcal{F}))\right)\right)[1] \\
\downarrow \lambda\left(\Psi_{2 n}^{r}\right) & & \lambda\left(\psi_{2 n}^{r+s}\right) \downarrow \\
\Omega_{U}^{2 n-\bullet} & \stackrel{\mathrm{id}}{\longrightarrow} & \Omega_{U}^{2 n-\bullet}
\end{array}
$$

Since $C_{\bullet}^{\text {lie }}\left(\Gamma\left(U, \mathfrak{g l}_{\infty}^{\mathrm{fin}}(\mathcal{D} \operatorname{iff}(\mathcal{E}))\right)\right)[1]$ is dense in $\widehat{\mathrm{C}}_{\bullet}^{\text {lie }}\left(\Gamma\left(U, \mathfrak{g l}_{\infty}^{\mathrm{fin}}(\mathcal{D} \operatorname{iff}(\mathcal{E}))\right)\right)[1]$ (and similarly for $\left.\mathcal{E} \oplus \mathcal{F}\right)$, and since all the maps involved in diagram (19) are continuous, commutativity of diagram (18) follows (note that this "commutativity on the nose" of diagram (18) is with our convenient choice of section of $\left.J_{\infty} \mathcal{E}\right|_{U}$ over $\left.J_{1} \mathcal{E}\right|_{U}$ as well as our choice trivialization of $J_{1} \mathcal{E}$ over $U$ ). This proves the desired proposition.

We remark that our proof of Propositions 20, 21 and 22 go through for arbitrary complex parallelizable manifolds. Further, if $\Xi_{2 n+1}$ is replaced by a $G L(n) \times G L(r)$-basic cocycle having the same class in cohomology, Proposition 20 is replaced by the obvious analog of Proposition 12, which goes through for arbitrary $X$ and arbitrary $\mathcal{E}$ with bounded geometry. Similarly, if $\Psi_{2 n+1}$ is replaced by a $G L(n)$-basic cocycle representing the same cohomology class, Propositions 21 and 22 hold for arbitrary $X$ and arbitrary $\mathcal{E}$ and $\mathcal{F}$ with bounded geometry.

4.2.4. Proof of Theorem 3. For this subsection, we shall assume that $X$ is complex parallelizable, $\mathcal{E}$ is trivial and $\mathcal{E}$ has bounded geometry. The first two assumptions can be removed if $\Psi_{2 n+1}$ is replaced by a $G L(n)$-basic cocycle representing the same cohomology class. By Propositions 20 and 21, for any choice used in the construction of $\lambda\left(\Psi_{2 n+1}^{r}\right)$, the map

$$
\int_{X} \lambda\left(\Psi_{2 n+1}^{r}\right): \quad \Gamma_{c}(X, \widetilde{\operatorname{Lie}}(\mathcal{D i f f}(\mathcal{E}))[1]) \rightarrow \mathbb{C}, \quad \alpha \mapsto \int_{X} \lambda\left(\Psi_{2 n+1}^{r}\right)(\alpha)
$$

induces the same map on the 0 -th homology of $\Gamma_{c}(X, \widetilde{\operatorname{Lie}}(\mathcal{D} \operatorname{iff}(\mathcal{E}))[1])$. On the other hand, we saw in Section 2.4.2 that $I_{\mathrm{FLS}}^{\text {lie, } \mathcal{E}}$ induces a map on the 0 -th homology of $\Gamma_{c}(X, \widetilde{\operatorname{Lie}}(\mathcal{D} \operatorname{iff}(\mathcal{E}))[1])$ as well. By Corollary $1, \mathrm{H}_{0}\left(\Gamma_{c}(X, \widetilde{\operatorname{Lie}}(\mathcal{D} \operatorname{iff}(\mathcal{E}))[1])\right)$ is a 1-dimensional $\mathbb{C}$-vector space. It follows that as linear functionals on $\mathrm{H}_{0}\left(\Gamma_{c}(X, \widetilde{\operatorname{Lie}}(\mathcal{D} \operatorname{iff}(\mathcal{E}))[1])\right)$,

$$
\int_{X} \lambda\left(\Psi_{2 n+1}^{r}\right)=C(X, \mathcal{E}) \cdot I_{\mathrm{FLS}}^{\mathrm{lie}, \mathcal{E}}
$$

Propositions 7 and 22 together with the nontriviality of $I_{\mathrm{FLS}}^{\text {lie, } \mathcal{E}}$ on homology imply that $C(X, \mathcal{E})=$ $C(X, \mathcal{E} \oplus \mathcal{F})$ for any vector bundle $\mathcal{F}$ with bounded geometry on $X$. This shows that $C(X, \mathcal{E})$ is independent of $\mathcal{E}$.

For the rest of this subsection assume that $\mathcal{E}=\mathcal{O}_{X}$. Let $U$ be an open subset of $X$ with holomorphic coordinates that identify $U$ with an open disk in $\mathbb{C}^{n}$. Choose a nontrivial 0-cycle $\alpha$ of $\Gamma_{c}(U, \widetilde{\operatorname{Lie}}(\mathcal{D} \operatorname{iff}(\mathcal{E}))[1])$. Note that after making the necessary choices, the construction of $\lambda\left(\Psi_{2 n+1}\right)$ is local in nature. It follows that

$$
\int_{U} \lambda\left(\Psi_{2 n+1}\right)(\alpha)=\int_{X} \lambda\left(\Psi_{2 n+1}\right)\left(j_{*} \alpha\right)
$$

where $j: U \rightarrow X$ is the natural inclusion. 
Further, if $\phi$ is an element of $\operatorname{Diff}^{0}(X)$ that is compactly supported on $U$,

$$
\operatorname{str}\left(\phi e^{-t \Delta_{X}}\right)=\operatorname{str}\left(\phi e^{-t \Delta_{U}}\right)
$$

for any $t>0$. In the right hand side of the above equation, we think of $\phi$ and $e^{-t \Delta_{U}}$ as endomorphisms of the space of square integrable sections of $\mathrm{K}^{\bullet} \mathcal{O}_{U}$. To see this, note that if $p_{t}(x, y)$ is the kernel of $e^{-t \Delta_{X}}$,

$$
\operatorname{str}\left(\phi e^{-t \Delta_{X}}\right)=\int_{X} \operatorname{str}\left(\phi p_{t}(x, x)\right)|d x|=\int_{U} \operatorname{str}\left(\phi p_{t}(x, x)\right)|d x|=\operatorname{str}\left(\phi e^{-t \Delta_{U}}\right) .
$$

The second equality above is because $\phi$ is compactly supported on $U$. The third equality above is because the heat kernel on $U$ is unique once the choice of Hermitian metric on $\mathrm{K}^{\bullet}{ }_{\mathcal{O}_{U}}$ is fixed (see [9]). It follows from equation (20) (in the case when $\mathcal{E}=\mathcal{O}_{X}$ ) and equation (21) that $C(X, \mathcal{E})$ is independent of $X$ as well. This proves Theorem 3.

4.2.5. For this subsection, let $X$ be an arbitrary compact smooth manifold. Let $\mathcal{F}_{\bullet}$ be a complex of sheaves on $X$ such that each $\mathcal{F}_{i}$ is a module over the sheaf of smooth functions on $X$. Suppose also that $\mathcal{F}_{\bullet}$ is in $\mathrm{D}^{b}\left(\mathrm{Sh}_{\mathbb{C}}[X]\right)$. Let $\mathfrak{U}:=\left\{U_{i}\right\}$ be a finite good cover of $X$. Consider the double complex $\check{C}\left(\mathfrak{U}, \mathcal{F}_{\bullet}\right)$ where

$$
\check{C}_{p, q}\left(\mathfrak{U}, \mathcal{F}_{\bullet}\right)=\oplus_{j_{1}<\cdots<j_{p}} \Gamma_{c}\left(U_{j_{1}} \cap \cdots \cap U_{j_{p}}, \mathcal{F}_{q}\right) .
$$

Here, $\Gamma_{c}$ denotes "sections with compact support". The vertical differential in this double complex is induced by the differential on $\mathcal{F}_{\bullet}$. The horizontal differential $\delta$ is given by the formula

$$
(\delta \omega)_{j_{1}, \ldots, j_{p-1}}=\sum_{j_{0}=1}^{N}(\omega)_{j_{0}, \ldots, j_{p-1}} .
$$

Here, $(\alpha)_{i_{1}, \ldots, i_{k}}$ denotes the component of a chain $\alpha \in \check{C}_{k, l}\left(\mathfrak{U}, \mathcal{F}_{\bullet}\right)$ in $\Gamma_{c}\left(U_{i_{1}} \cap \cdots \cap U_{i_{k}}, \mathcal{F}_{l}\right)$. We also follow the convention that if $i_{1}<\cdots<i_{k},(\alpha)_{i_{\sigma(1)}, \ldots, i_{\sigma(k)}}=\operatorname{sgn}(\sigma)(\alpha)_{i_{1}, \ldots, i_{k}}$ for any permutation $\sigma$ of $1, \ldots, k$. The following proposition is a trivial modification of Proposition 12.12 of Bott and $\mathrm{Tu}[3]$. We therefore, omit its proof.

\section{Proposition 23.}

(1) The map

$$
\operatorname{Tot}_{\bullet}\left(\check{C}\left(\mathfrak{U}, \mathcal{F}_{\bullet}\right)\right) \rightarrow \Gamma_{c}\left(X, \mathcal{F}_{\bullet}\right), \quad \alpha \mapsto \sum_{i}(\alpha)_{i}
$$

is a quasi-isomorphism.

(2) $\mathrm{H}_{p}\left(\operatorname{Tot}_{\bullet}\left(\check{C}\left(\mathfrak{U}, \mathcal{F}_{\bullet}\right)\right)\right) \simeq \mathbb{H}_{c}^{-p}\left(X, \mathcal{F}_{\bullet}\right)$.

The subscript ' $c$ ' on the right hand side in this proposition stands for "compact support". The chain complex $\mathcal{F}_{\bullet}$ needs to be converted into a cochain complex by inverting degrees in order to make sense of the hypercohomology in the above proposition.

Let $\alpha$ be 0 -cycle in $\Gamma_{c}\left(X, \mathcal{F}_{\bullet}\right)$.

Proposition 24. Suppose that $\mathcal{F}_{\bullet}$ is acyclic in negative degrees. If $X$ is compact, there exist 0 -cycles $\alpha_{i}$ of $\Gamma_{c}\left(U_{i}, \mathcal{F}_{\bullet}\right)$ such that $\sum_{i}\left[\alpha_{i}\right]=[\alpha]$ in $\mathrm{H}_{0}\left(\Gamma_{c}\left(X, \mathcal{F}_{\bullet}\right)\right)$.

Proof. We use a "staircase argument". By Proposition 23, there exists a 0-cycle $\hat{\alpha}$ of $\operatorname{Tot}_{\bullet}\left(\check{C}\left(\mathfrak{U}, \mathcal{F}_{\bullet}\right)\right)$ such that $[\hat{\alpha}]$ maps to $[\alpha]$ under the quasi-isomorphism in Proposition 23. Note that $\hat{\alpha}=\sum_{k \geq 1} \hat{\alpha}_{k}$ where $\hat{\alpha}_{j}$ is a $-k+1$-chain in $\oplus_{j_{1}<\cdots<j_{k}} \Gamma_{c}\left(U_{j_{1}} \cap \cdots \cap U_{j_{k}}, \mathcal{F}_{\bullet}\right)$. Let $N(\hat{\alpha})$ 
be the largest integer such that $\hat{\alpha}_{N(\hat{\alpha})} \neq 0$. Then, $\hat{\alpha}_{N(\hat{\alpha})}$ is a cycle in $\oplus_{j_{1}<\cdots<j_{k}} \Gamma_{c}\left(U_{j_{1}} \cap\right.$ $\left.\cdots \cap U_{j_{N(\hat{\alpha})}}, \mathcal{F}_{\bullet}\right)$. Since, $\mathcal{F}_{\bullet}$ is acyclic in negative degrees, $\hat{\alpha}_{N(\hat{\alpha})}=\delta \beta$ for some $\beta$ where $\delta$ is the differential on $\oplus_{j_{1}<\cdots<j_{k}} \Gamma_{c}\left(U_{j_{1}} \cap \cdots \cap U_{j_{N(\hat{\alpha})}}, \mathcal{F}_{\bullet}\right)$. Let $d$ denote the differential in the bicomplex $\operatorname{Tot}_{\bullet}\left(\check{C}\left(\mathfrak{U}, \mathcal{F}_{\bullet}\right)\right)$. View $\beta$ as a chain in $\operatorname{Tot}_{\bullet}\left(\check{C}\left(\mathfrak{U}, \mathcal{F}_{\bullet}\right)\right)$. Then, $\hat{\alpha}+d \beta$ is homologous to $\hat{\alpha}$. On the other hand, $N(\hat{\alpha}+d \beta)=N(\hat{\alpha})-1$. It follows that repeating this procedure eventually gives a 0 -cycle in $\oplus_{i} \Gamma_{c}\left(U_{i}, \mathcal{F}_{\bullet}\right)$ homologous to $\hat{\alpha}$. This proves the desired proposition.

4.2.6. Shoikhet's holomorphic noncommutative residue: definition. Let $X$ be an arbitrary compact complex manifold and let $\mathcal{E}$ be an arbitrary vector bundle on $X$. Let $\mathfrak{U}:=\left\{U_{i}\right\}$ be a finite good cover of $X$. Let $\mathcal{D}$ be a global holomorphic differential operator on $\mathcal{E}$. Note that $\mathrm{E}_{11}(\mathcal{D})$ is a 0 -cycle in $\Gamma_{c}(X, \widetilde{\operatorname{Lie}}(\mathcal{D} \operatorname{iff}(\mathcal{E}))[1])$. Applying Proposition 24 to the complex $\widetilde{\operatorname{Lie}}(\mathcal{D} \operatorname{iff}(\mathcal{E}))[1]$ of sheaves on $X$, we see that there exist 0 -cycles $\alpha_{i}$ in $\Gamma_{c}\left(U_{i}, \widetilde{\operatorname{Lie}}(\mathcal{D} \operatorname{iff}(\mathcal{E}))[1]\right)$ such that $\sum_{i}\left[\alpha_{i}\right]=\left[\mathrm{E}_{11}(\mathcal{D})\right]$ in $\mathrm{H}_{0}\left(\Gamma_{c}(X, \widetilde{\operatorname{Lie}}(\mathcal{D} \operatorname{iff}(\mathcal{E}))[1])\right)$. Define the holomorphic noncommutative residue of $\mathcal{D}$ to be the sum

$$
\mathrm{NC}(\mathcal{D}):=\sum_{i} \int_{U_{i}} \lambda\left(\Psi_{2 n+1}^{r}\right)\left(\alpha_{i}\right)
$$

One can make the choices used to define $\lambda\left(\Psi_{2 n+1}^{r}\right)$ in the $U_{i}$ arbitrarily. We also remark that $\lambda\left(\Psi_{2 n+1}^{r}\right)$ is not globally defined on $X$ in a direct way (at least as far as we can see). By Theorem 3 ,

$$
\begin{aligned}
\mathrm{NC}(\mathcal{D}) & =\sum_{i} \int_{U_{i}} \lambda\left(\Psi_{2 n+1}^{r}\right)\left(\alpha_{i}\right)=C \cdot \sum_{i} I_{\mathrm{FLS}}^{\mathrm{lie}, \mathcal{E}}\left(\alpha_{i}\right)=C \cdot I_{\mathrm{FLS}}^{\mathrm{lie}, \mathcal{E}}\left(\sum_{i} \alpha_{i}\right) \\
& =C \cdot I_{\mathrm{FLS}}^{\mathrm{lie}, \mathcal{E}}\left(\mathrm{E}_{11}(\mathcal{D})\right)=C . \lim _{t \rightarrow \infty} \operatorname{str}\left(\mathcal{D} e^{-t \Delta_{\mathcal{E}}}\right)=C \cdot \operatorname{str}(\mathcal{D}) .
\end{aligned}
$$

This proves Conjecture 3.3 of [20] in greater generality.

Alternatively, one can sidestep the complications that arose while defining $\mathrm{NC}(\mathcal{D})$ as follows. Let $\Theta_{2 n+1}$ be a $G L(n)$-basic cocycle representing the cohomology class $\left[\Psi_{2 n+1}\right]$ (its existence is proven following the proof of Lemma 5.2 in [10]). Construct the cocycles $\Theta_{2 n+1}^{r}$ from $\Theta_{2 n+1}$ exactly as the $\Psi_{2 n+1}^{r}$ were constructed from $\Psi_{2 n+1}$. The cocycles $\Theta_{2 n+1}^{r}$ are $G L(n) \times G L(r)$ basic representatives of the class $\left[\Psi_{2 n+1}^{r}\right]$. Then, $\lambda\left(\Theta_{2 n+1}^{r}\right)$ is a globally defined $2 n$-form and define

$$
\mathrm{NC}(\mathcal{D}):=\int_{X} \lambda\left(\Theta_{2 n+1}^{r}\right)\left(\mathrm{E}_{11}(\mathcal{D})\right)
$$

The two definitions of $\mathrm{NC}(\mathcal{D})$ given here are equivalent by Proposition 19.

\section{Acknowledgements}

I am grateful to Giovanni Felder and Thomas Willwacher for some very useful discussions. This work would not have reached its current form without their pointing out important shortcomings in earlier versions. I am also grateful to Boris Shoikhet for useful discussions. I thank the referees of this article for their constructive suggestions. This work was done (prior to my joining my current position) partly at Cornell University and partly at IHES. I am grateful to both these institutions for providing me with a congenial work atmosphere. 


\section{References}

[1] Adler M., On a trace functional for formal pseudo differential operators and the symplectic structure of the Korteweg-de Vries type equations, Invent. Math. 50 (1979), 219-248.

[2] Berline N., Getzler E., Vergne M., Heat kernels and Dirac operators, Springer-Verlag, Berlin, 2004.

[3] Bott R., Tu L.W., Differential forms in algebraic topology, Springer-Verlag, Berlin, 1995.

[4] Bressler P., Kapranov M., Tsygan B., Vasserot E., Riemann-Roch for real varieties, in Algebra, Arithmetic and Geometry: in Honor of Yu.I. Manin, Progr. Math., Vol. 269, Birkhäuser Boston, Inc., Boston, MA, 2009, 125-164, math.DG/0612410.

[5] Brylinski J.-L., A differential complex for Poisson manifolds, J. Differential Geom. 28 (1988), 93-114.

[6] Calaque D., Dolgushev V., Halbout G., Formality theorems for Hochschild chains in the Lie algebroid setting, J. Reine Angew. Math. 612 (2007), 81-127, math.KT/0504372.

[7] Calaque D., Rossi C., Lectures on Duflo isomorphisms in Lie algebras and complex geometry, Lecture notes, available at http://math.univ-lyon1.fr/ calaque/LectureNotes/LectETH.pdf.

[8] Dolgushev V., Covariant and equivariant formality theorems, Adv. Math. 191 (2005), 147-177, math.QA/0307212.

[9] Donelly H., Asymptotic expansions for the compact quotients of properly discontinuous group actions, Illinois J. Math. 23 (1979), 485-496.

[10] Engeli M., Felder G., A Riemann-Roch-Hirzebruch formula for traces of differential operators, Ann. Sci. Éc. Norm. Supér. (4) 41 (2008), 621-653, math.QA/0702461.

[11] Feigin B.L., Felder G., Shoikhet B., Hochschild cohomology of the Weyl algebra and traces in deformation quantization, Duke Math. J. 127 (2005), 487-517, math.QA/0311303.

[12] Feigin B.L., Tsygan B.L., Cohomology of the Lie algebra of generalized Jacobian matrices, Funktsional. Anal. i Prilozhen. 17 (1983), no. 2, 86-87 (in Russian).

[13] Gel'fand I.M., Fuks D.B., Cohomology of the Lie algebra of tangent vector fields on a smooth manifold. II, Funktsional. Anal. i Prilozhen. 4 (1970), no. 2, 23-31 (in Russian).

[14] Kashiwara M., Schapira P., Sheaves on manifolds, Springer-Verlag, Berlin, 2002.

[15] Loday J.-L., Cyclic homology, Springer-Verlag, Berlin, 1992.

[16] Nest R., Tsygan B., Deformations of symplectic Lie algebroids, deformations of holomorphic symplectic structures, and index theorems, Asian J. Math. 5 (2001), 599-635, math.QA/9906020.

[17] Pflaum M.J., Posthuma H.B., Tang X., An algebraic index theorem for orbifolds, Adv. Math. 210 (2007), 83-121, math.KT/0507546.

[18] Ramadoss A.C., Some notes on the Feigin-Losev-Shoikhet integral conjecture, J. Noncommut. Geom. 2 (2008), 405-448, math.QA/0612298.

[19] Ramadoss A.C., Integration over complex manifolds via Hochschild homology, J. Noncommut. Geom. 3 (2009), 27-45, arXiv:0707.4528.

[20] Shoikhet B., Integration of the lifting formulas and the cyclic homology of the algebra of differential operators, Geom. Funct. Anal. 11 (2001), 1096-1124, math.QA/9809037.

[21] Shoikhet B., Lifting formulas. II, Math. Res. Lett. 6 (1999), 323-334, math.QA/9801116.

[22] Willwacher T., Cyclic cohomology of the Weyl algebra, arxiv:0804.2812.

[23] Wodzicki M., Cyclic homology of differential operators, Duke Math. J. 54 (1987), 641-647. 